\title{
Explaining the competitive advantage generated from Analytics with the knowledge-based view: the example of Logistics and Supply Chain Management
}

\author{
Tino T. Herden ${ }^{1}(D$
}

Received: 11 February 2019/Accepted: 14 November 2019/Published online: 11 December 2019

(C) The Author(s) 2019

\begin{abstract}
The purpose of this paper is to provide a theory-based explanation for the generation of competitive advantage from Analytics and to examine this explanation with evidence from confirmatory case studies. A theoretical argumentation for achieving sustainable competitive advantage from knowledge unfolding in the knowledge-based view forms the foundation for this explanation. Literature about the process of Analytics initiatives, surrounding factors, and conditions, and benefits from Analytics are mapped onto the knowledge-based view to derive propositions. Eight confirmatory case studies of organizations mature in Analytics were collected, focused on Logistics and Supply Chain Management. A theoretical framework explaining the creation of competitive advantage from Analytics is derived and presented with an extensive description and rationale. This highlights various aspects outside of the analytical methods contributing to impactful and successful Analytics initiatives. Thereby, the relevance of a problem focus and iterative solving of the problem, especially with incorporation of user feedback, is justified and compared to other approaches. Regarding expertise, the advantage of cross-functional teams over data scientist centric initiatives is discussed, as well as modes and reasons of incorporating external expertise. Regarding the deployment of Analytics solutions, the importance of consumability, users assuming responsibility of incorporating solutions into their processes, and an innovation promoting culture (as opposed to a data-driven culture) are described and rationalized. Further, this study presents a practical manifestation of the knowledge-based view.
\end{abstract}

Electronic supplementary material The online version of this article (https://doi.org/10.1007/s40685019-00104-x) contains supplementary material, which is available to authorized users.

Tino T. Herden

herden@logistik.tu-berlin.de

1 Berlin Institute of Technology, Straße des 17. Juni 135, 10623 Berlin, Germany 
Keywords Analytics - Data science - Logistics - Supply chain management · Knowledge-based view

\section{Introduction}

The use of Analytics is increasing across industries. It is fueled by trending concepts like big data and data science, innovative technologies such as distributed computing and in-memory databases, as well as the rapid increase of data available for processing. A recent survey showed a constant increase of organizations perceiving the role of Analytics as critical, a closing maturity and capability gap between digital natives and traditional companies in applying Analytics and its strategic role, as embodied in appointments of C-level executives for Analytics (Alles and Burshek 2016). A large proportion of surveye dorganizations believe that they can gain competitive advantages from [employing] Analytics. Another study goes as far as to suggest that data-empowered organizations may threaten the market survival of companies not using these approaches (Capgemini 2015). However, organizations also struggle with adapting Analytics successfully (Viaene and Van den Bunder 2011), with one difficulty being the ongoing discussion about what defines Analytics. Holsapple et al. (2014) investigated a plethora of definitions of Analytics, including the definition by Davenport and Harris (2007) who initiated the broader recognition of Analytics with their famous book. Holsapple et al. (2014) identified at least six definitional perspectives on Analytics just in the literature they reviewed, highlighting the diverse comprehension of the topic. Based on the core characteristics of Analytics, it has been described as recognizing and solving business problems based on evidence such as data, facts, but also well-reasoned estimations. Further, analytic initiatives are diverse and have to fit with people, processes, and tasks to enable their benefits (Ghasemaghaei et al. 2017), demanding investigation of which practices and conditions lead to generation of competitive advantage from Analytics.

Competitive advantage is frequently discussed in the strategic management literature. The resource-based view argues for competitive advantage based on the resources of firms, including assets, capabilities, processes, attributes, and knowledge-if these are rare, imperfectly imitable, and non-substitutable (Barney 1991). The capability-based view emphasizes these resources as the capabilities of firms that cannot be purchased on the market and require strategic vision to develop over time through the strategic decisions of bounded rational managers facing uncertainty, complexity, and conflict (Amit and Schoemaker 1993). The relational view argues that firms' resources are of limited value in providing competitive advantage and instead credit it to the combined resources of a network of firms (Dyer and Singh 1998). Finally, the knowledge-based view narrows down the resource required to provide competitive advantage to firms to just one item, which satisfies all the necessary characteristics - the knowledge held by the individuals of the firm (Grant 1996a). Managers are responsible for integrating and applying that knowledge. As the integration and application process of knowledge fits the definition of Analytics as problem recognition and solving, the knowledge-based 
view provides a reasonable theoretical grounding to investigate the generation of competitive advantage from Analytics.

One discipline increasingly adapting Analytics is Logistics and Supply Chain Management (LSCM). Scholars expect Analytics to change how supply chains operate (Schoenherr and Speier-Pero 2015). In practice, executives assess Analytics as playing a pivotal role in driving profit and creating competitive advantage in LSCM (Thieullent et al. 2016). Due to the vast number of applications areas and the assumed potential, a sub-discipline of Analytics used in LSCM has formed labeled SCM data science (Waller and Fawcett 2013) or supply chain Analytics (Chae et al. 2014a; Souza 2014). LSCM is considered an early adopter of analytical methods, using operations research to optimize inventories, locations, and transportation costs (Davenport 2009). Holsapple et al. (2014) even cited an article on production control and automation, while exhibiting the origins of Analytics. In recent research, the use of Analytics has shown a positive impact on LSCM performance (Trkman et al. 2010; Sanders 2016; Chavez et al. 2017) and researchers have called for further research on Analytics in LSCM (Waller and Fawcett 2013; Schoenherr and Speier-Pero 2015). However, research has also shown that a major proportion of organizations remains reluctant to use Analytics or are not even familiar with it, due to, amongst other factors, lack of ideas about how to achieve advantage from it (Schoenherr and Speier-Pero 2015; Sanders 2016).

To investigate Analytics' impact on organizations' competitive advantage, narrowing the focus is necessary. This article's investigation focuses on the example of LSCM for several reasons in addition to the field being an early adopter and achieving considerable value from employing Analytics. From its core characteristics, LSCM is driven by efficiency and cost effectiveness (Simchi-Levi et al. 2003), which demands sophisticated decision making-as supported by Analytics. Therefore, it is not surprising that LSCM has a long history of emphasizing data-driven decision making (Waller and Fawcett 2013; Souza 2014). LSCM is usually a complex task, managing information, products, services, and financial and knowledge flows across internal units such as procurement and manufacturing, as well as between globally dispersed organizations, including suppliers, retailers, or manufacturers (Bowersox et al. 2007). Consequently, collaborative approaches to Analytics are needed, presenting a unique challenge for Analytics, since data come from several different organizations and results are deployed across them (Davenport 2009). In addition, LSCM is a human-centered process with a variety of decision makers acting on the basis of their personal experience, resulting in unexpected events, human errors, and consequential dynamic effects in the processes (Wang et al. 2014). Wang et al. (2014) further highlighted the diversity of processes and the resulting heterogeneity of process knowledge. In summary, LSCM is chosen as a focus due to the field's experience with data-driven solutions, the constant demand for further improvement, and the challenges associated with adopting Analytics given the complex, diverse, dispersed, and error-prone processes distributed across several business units, organizations, and decision makers.

Considering the impact of Analytics, the objective of this research is to investigate how organizations can generate competitive advantage from Analytics. 
For this purpose, Analytics' dependency as inputs not only on data, but also domain knowledge, is acknowledged (Provost and Fawcett 2013), consequently leading to activities in a specific domain like LSCM being more similar to each other as compared to activities in a different domain. In research, this results in domainisolated chains of references, since scholars tend to refer scholars from the same domain (Holsapple et al. 2014). Thus, to create coherence of research and to narrow the focus, empirical evidence will be collected from organizations maturely employing Analytics in a LSCM context. This leads to the following research question: how is competitive advantage generated from Analytics in context of Logistics and Supply Chain Management?

The remainder of this article is structured as follows. Section 2 reviews the knowledge-based view and literature on Analytics to establish a link between them, as embodied in the propositions formulated for this research. Section 3 documents the research design, for which confirmatory case studies have been chosen. Section 4 discusses the results, with a focus on the proposition of creating an extensive explanation of how Analytics generates competitive advantage. The research will be concluded in Sect. 5, which provides implications, limitations, and indications for future research.

\section{Theoretical background}

In this section, Analytics activities are mapped onto the argumentation for competitive advantage from the knowledge-based view (KBV) to present their congruence. The connecting points are summarized in ten propositions.

\subsection{Knowledge-based view}

The KBV explains the generation of sustainable competitive advantage from knowledge, summarized in the following section. Regarding knowledge, its creation, transfer, and integration are distinguished as follows: creation refers to development of new knowledge. Transfer indicates sharing of knowledge without implying that the receiver gains the ability to apply it. In contrast, knowledge integration describes the sharing of knowledge such that receivers can apply it, but without necessarily possessing it.

\subsubsection{The source of knowledge-based competitive advantage}

An organization ("firm") cannot use the open market as a source of sustainable competitive advantage (Barney 1986, 1991). Instead, according to the resourcebased view, it must create such advantage from its resources, which need to be rare, imperfectly imitable, and non-substitutable.

Developing from the resource-based view, scholars have created several other argumentations. These include the $\mathrm{KBV}$, which establishes the knowledge possessed by organizations as their most essential resource for competitive advantage (Conner and Prahalad 1996; Grant 1996a, b). It is based on an emphasis 
of the strategical value of knowledge in organizations (Teece 1981; Winter 1987; Kogut and Zander 1992) and differentiating the performance of organizations using asymmetries in knowledge (Conner and Prahalad 1996). In the KBV, the role of individuals is underlined. Organizational members carry, generate, and preserve the knowledge, while integration of knowledge for its application is governed by managers of organizations. Neither holding knowledge without integration nor the attempt to integrate non-existing knowledge can be a source of competitive advantage (Grant 1996b). Thus, the KBV also deals with issues of organizational coordination and structure (Grant 1996a).

The aspect most essential for the knowledge integration and application is the degree of its transferability (Grant 1996a), which depends on its form: explicit or tacit (Winter 1987; Kogut and Zander 1992; Grant 1996a; Teece 1998). Perfectly explicit knowledge is easy to articulate and communicate, transmittable without loss of integrity, and observable, consumable, learnable, and usable with insignificant marginal costs (Winter 1987; Kogut and Zander 1992; Grant 1996a). In contrast, perfectly tacit knowledge is difficult to articulate; not completely transferable in substance and meaning; and costly to transfer, since it is tied to skills and experience-based intuition, revealed by application and acquired through practice ("know-how") (Kogut and Zander 1992; Grant 1996b; Teece 1998; Nonaka and von Krogh 2009). These forms exist on a continuum indicating some degree of tacitness in almost all knowledge (Spender 1996). For organizations, this distinction implies the need for different actions to exploit the knowledge (Teece 1998).

Tacitness of knowledge is substantial in the argumentation for generating competitive advantage from knowledge unfolded by Grant (1996a, b), Spender (1996) and Teece (1998) and was followed repeatedly (Zack 1999; Alavi and Leidner 2001; Purvis et al. 2001; Vachon and Klassen 2008). Due to tacitness, knowledge as a resource can be scarce, non-transferable, and non-replicable. This denotation, adapted from the relevant conditions of the resource-based view, equally describes how access is denied to competitors. Organizations possess such knowledge, which resides in specialists who gain it through learning and knowledge creation (not further explained by scholars), but which cannot be communicated completely or easily and cannot be converted to utility without the support of other individuals (Demsetz 1988). Based on Demsetz (1988), the KBV argumentation suggests higher outcomes from several specialists combining their knowledge and rejects a "jack-of-all-trades". However, the knowledge must be put into action for competitive advantage. Thus, Spender (1996) and Grant (1996a, b) conclude that organizations' managers must guide organizational members to execute complex, team-based productive activities resulting in the combination of their knowledge, making it possible to apply that knowledge during the value creation process of transforming input to output. Thereby, the combination represents knowledge integration with inimitable organizational capabilities as the outcome.

For knowledge integration into the value creation process, Grant (1996a) identified four mechanisms that several scholars have elaborated (Heugens et al. 2004; Spanos and Prastacos 2004; Canonico et al. 2012; Hurnonen et al. 2016): (1) rules and directives, (2) sequencing, (3) routines, and (4) group problem solving and decision making. They aim to integrate the knowledge efficiently, defined as 
effectively integrating, while minimizing the transfer, which is dependent on process characteristics, division of tasks between individuals, and organizational design (Grant 1996a). These mechanisms, especially the first three, can be rather impersonal, communication minimizing, and knowledge transfer economizing ("automating" or "programming") (Ven et al. 1976; Grant 1996a; Alavi and Leidner 2001; Spanos and Prastacos 2004; Hurnonen et al. 2016). In contrast, lateral relationships that are personal, less straightforward, and interaction dependent can be deployed as in the fourth mechanism (Galbraith 1973; Grant 1996a; Canonico et al. 2012). In accordance with dependence on different process and organizational characteristics, mechanisms must be suitable for varying complexity, uncertainty, or importance of tasks (Grant 1996a; Spanos and Prastacos 2004; Hurnonen et al. 2016). Knowledge integration is illustrated in Fig. 1, with numbers referring to the propositions introduced later in this chapter.

The outcome of knowledge integration depends on the knowledge all individuals of an organization share due to their affiliation to the organization, since knowledge integration is catalyzed by this common knowledge (Grant 1996a, b; Spender 1996). An organization's internal knowledge, combining specialized and common knowledge with knowledge integration mechanisms, cannot be accessed by competitors. Thus, they are not able to use the same organizational capabilities and, hence, cannot achieve the same value created by tangible resources in the value creation process of transforming input to output. Provided these organizational capabilities are advantageous over competitors' capabilities, the competitive advantage depends on the efficiency of knowledge integration. The efficiency relies on (1) the level of common knowledge, (2) the frequency and variability of integration of common and specialized knowledge, and (3) structures that economize the communication needed for knowledge integration.

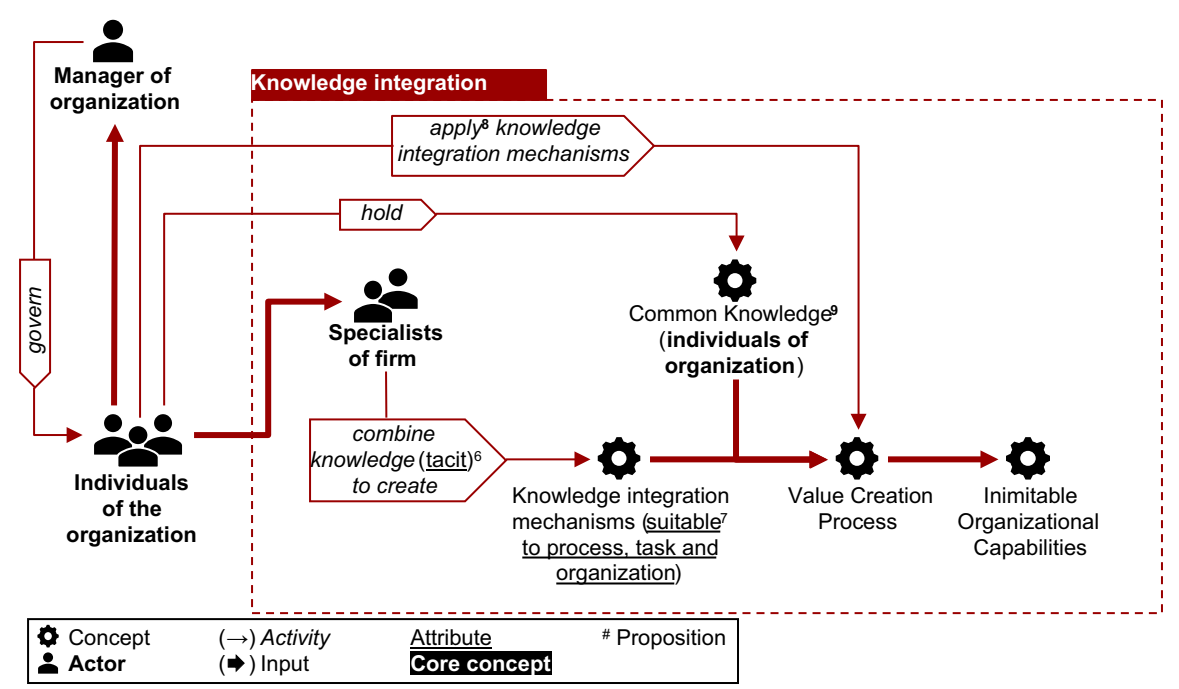

Fig. 1 Knowledge integration illustrated 
Grant (1996a, b) further discusses the conditions leading to sustainable competitive advantage. Its sustainability requires continuous renewal of organizational capabilities, accomplished by two actions: (1) extension of existing capabilities to include new types of knowledge and (2) use of existing knowledge in new capabilities. Solely executing either one of the actions may not be sufficient. As a result, the sustainability of the competitive advantage is dependent on increasing the scope of knowledge integration continuously. The conditions for sustainable competitive advantage are illustrated in Fig. 2.

\subsubsection{The problem-solving perspective on the knowledge-based view}

For the KBV, as described above, scholars both pointed out deficits and introduced extensions to cover originally unconsidered aspects. One of these aspects is the disregard for knowledge creation, which Grant (1996a) himself had already evaluated as serious. Nickerson and Zenger (2004) developed their extension to the $\mathrm{KBV}$ to cover this aspect based on solving valuable problems-subsequently referred to as the problem- solving perspective (PSP).

The PSP addresses two deficits of the KBV. First, the KBV does not explain efficient creation of knowledge. The PSP argues for solving valuable problems to create desired knowledge with value assessed by the expected value of the problem's solution and organizational capacity to profitably achieve the solution. Second, the market's irrelevance as a source of knowledge in the KBV is argued to be inaccurate in the PSP, resulting in the introduction of forms of solution search with varying levels of market inclusion. Scholars have also revisited the PSP with regard to managerial innovation, development, and improvement topics (Macher 2006; Hsieh et al. 2007; Tiwana 2008; Jeppesen and Lakhani 2010; Choo et al. 2015). Regarding these topics, knowledge creation must be organized efficiently and effectively by solving valuable problems, rather than exploiting existing knowledge. The value of a problem depends on the value that viable solutions can

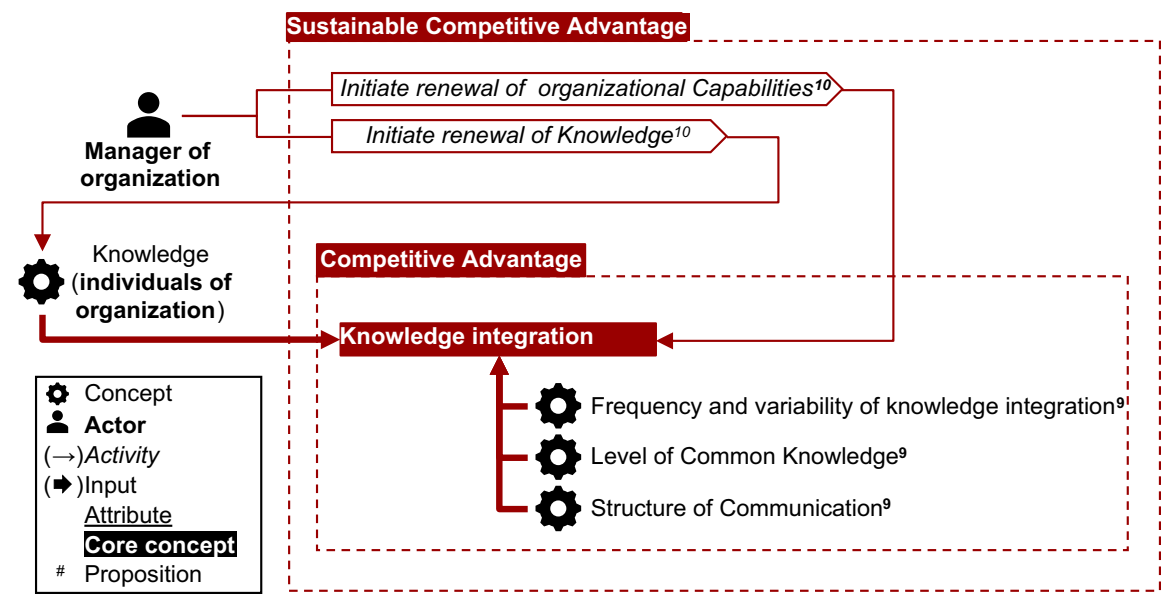

Fig. 2 Sustainable competitive advantage from the knowledge illustrated 
provide and the costs of identifying the solutions (Nickerson and Zenger 2004). Thereby, effectiveness and efficiency in problem solving result from generative problem solving based on learning and reflection (Choo et al. 2015). In contrast, symptomatic problem solving, which focuses on controlling the potential of negative outcomes- "fixing" a problem-do not contribute to knowledge creation and may affect existing knowledge negatively.

The value of problems and their solutions in the PSP builds on Simon's (1962, 1973) study of complex systems. He argues for a problem's generic concept being a goal state that is produced from an initial state (Simon 1962) and solving being a sequence of processes that must be discovered to get from initial state to goal state. The complexity of problems increases with increasing number of partsknowledge sets needed for the solution (Jeppesen and Lakhani 2010) — that interact in a non-simple way. Complex problems possess characteristics providing potentially valuable solutions and are resultingly valuable (Nickerson and Zenger 2004). Further, problems vary by the definition of their structure. This definition represents an abstract degree of how well the nature, substance or patterns of a problem are understood and known (Simon 1962). The definition of a problem locates it on a continuum between an ill-structured and well-structured state (Simon 1973). A problem is well structured if the practical effort for solution search is allowed by the existence of a problem space in which goal state, current states, attainable state changes, and acquirable knowledge about the problem including a reflection of external links can be represented with complete accuracy. Existence implies access to relevant knowledge sets on the structure due to knowledge possession or transfer, although this may be limited by the knowledge's tacitness. The development of a problem definition-discovering the various parts of the problem space-is argued to be the solution search (Simon 1973). Once the definition applicable to a problem is complete, it can be used for other occurrences of the same problem, because unknown knowledge set interactions are revealed and mastered such that the ill-structured problem changes to a well-structured one and [the approach of] how to solve it is understood (Jeppesen and Lakhani 2010; Macher and Boerner 2012).

The solution search consists of selective trials which uniquely combine chosen knowledge and which are distinguished by their directional nature and heuristics (Simon 1962; Nickerson and Zenger 2004). Directional search uses prior experience about positive and negative outcomes and reinforces the search in the direction leading to positive outcomes- "trial-and-error"-which is more suited for low knowledge set interactions, since it is prone to remain enclosed in a local solution area (Gavetti and Levinthal 2000; Nickerson and Zenger 2004; Hsieh et al. 2007). In contrast, heuristics are premised on beliefs about linkages between choices and actions, resulting in fewer trials and selection of relevant knowledge sets based on anticipated interaction, which is more suited for problems with high knowledge set interactions, but also requires increased knowledge transfer (Gavetti and Levinthal 2000; Nickerson and Zenger 2004). The knowledge creation process is illustrated in Fig. 3.

To execute the ideal solution search, the relevant knowledge sets' interactions need to be identified. Identifying the non-simple knowledge set interactions for 


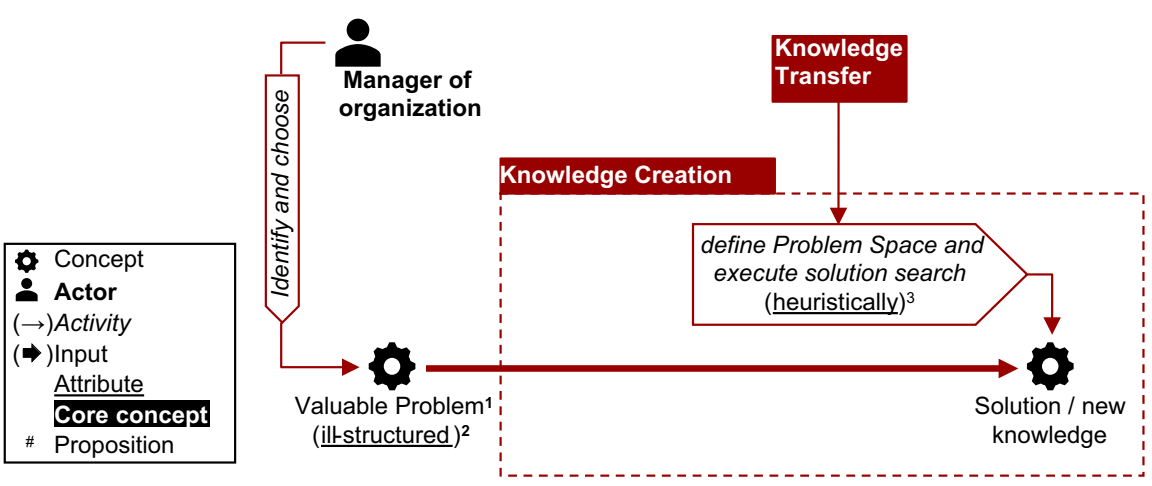

Fig. 3 Knowledge creation illustrated

solution search requires a group of individuals holding relevant specialized knowledge, which develops common cognitive maps limited by human cognitive constraints (Nickerson and Zenger 2004). The likelihood of creating new knowledge from combining knowledge sets increases with the diversity of the knowledge sets, while the difficulty of knowledge transfer also increases (Tiwana 2008; Jeppesen and Lakhani 2010). A mediator bringing some degree of knowledge set redundancy to the group could enhance knowledge brokering, translation, and interpretation, but has been argued to provide no benefit beyond that (Tiwana 2008). Further, marginal knowledge sets, which are distant from conventional approaches to a problem but close enough to have insight on its problem space, can be beneficial to the solution search by introducing approaches that are unconventional to the rest of the group (Jeppesen and Lakhani 2010). Assessment of both the ideal solution search and solution is the task of the manager, including gaining understanding of the problem space, identifying relevant knowledge sets and selecting a group of their holders, and choosing a governance mode for the solution search, leading to eventual knowledge acquisition (Nickerson and Zenger 2004).

The PSP describes three modes of governance, which vary in control over the solution search in terms of centralization and market inclusion and extent of knowledge transfer (Nickerson and Zenger 2004). Costs for knowledge transfer are higher for solution searches outsourced to the market, since the community within organizational boundaries can create a common communication framework, which can even enhance knowledge creation for ill-structured problems (Nickerson and Zenger 2004; Macher 2006; Hsieh et al. 2007; Macher and Boerner 2012). Hence, the market approach is suited for well-structured problems with lower knowledge transfer but higher chance of exploiting a more efficient incentive system. However, it also creates access to more diverse knowledge sets and new solutions with superior performance, justifying higher costs (Jeppesen and Lakhani 2010). In contrast, the knowledge transfer advantages of internal solution search are more suited for ill-structured problems. The sources of knowledge sets and their transfer are illustrated in Fig. 4. 


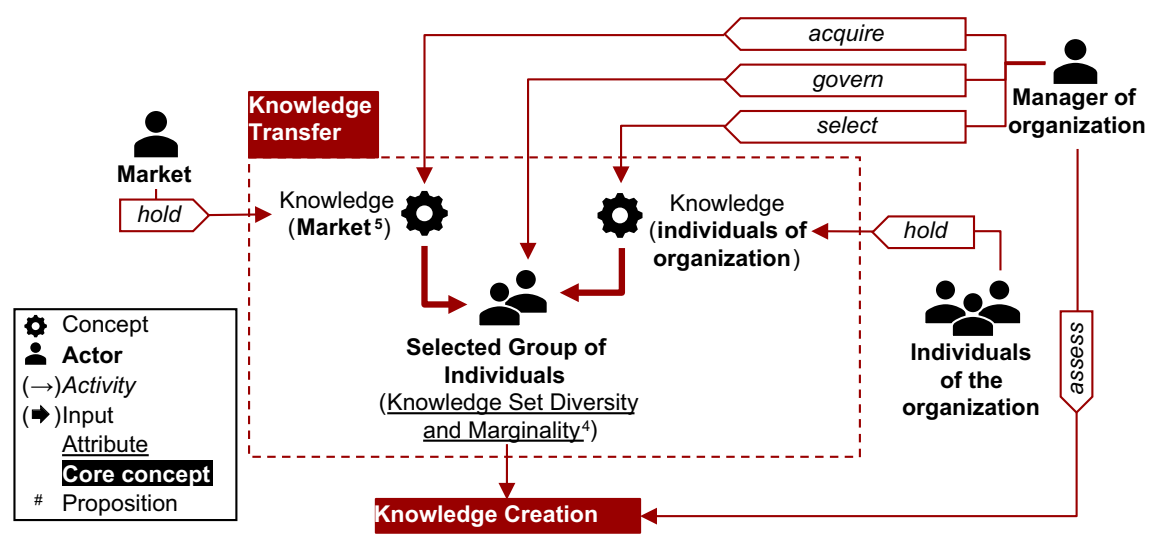

Fig. 4 Knowledge sources and transfer illustrated

\subsubsection{Synopsis of the knowledge-based view}

Nickerson and Zenger (2004) theorize that an organizational manager's generation of valuable solutions to valuable problems can be expected to, for example, enhance and develop products and services and reduce costs of production or delivery. Hence, they represent novel knowledge that can be combined with existing knowledge and integrated into the value creating transformation process of inputs to outputs, leading to a more advantageous output. To facilitate this integration, managers must assess the value of the solution and realize its integration with mechanisms described in the originating KBV. Hence, the PSP extends the KBV, which describes the integration of knowledge to generate competitive advantages, by explaining the creation of the necessary knowledge. The KBV and the PSP are illustrated in Fig. 5.

\subsection{Analytics}

Analytics creates knowledge, makes it usable, and benefits from it, supposedly resulting in a competitive edge (Davenport and Harris 2007). Thus, the KBV and Analytics show a high degree of agreement at the macro-level. The following section explores aspects on which the KBV and Analytics agree on a more granular level.

While the concept of Analytics is still subject to debate, similar to Larson and Chang (2016) and Holsapple et al. (2014), this study acknowledges related concepts such as business intelligence, big data, and data science as intertwined with Analytics. A vague distinction of Analytics is being focused on decision support in business processes, business intelligence being either an overarching term or the provision of information, big data being a technological advancement, and data science being advanced models and algorithms, but this distinction cannot be applied consistently and each concept draws from or depends on the others. With the decision support focus, which demands the integration of analytical insight into a process, Analytics display the most relevant and leading concept for this research. 


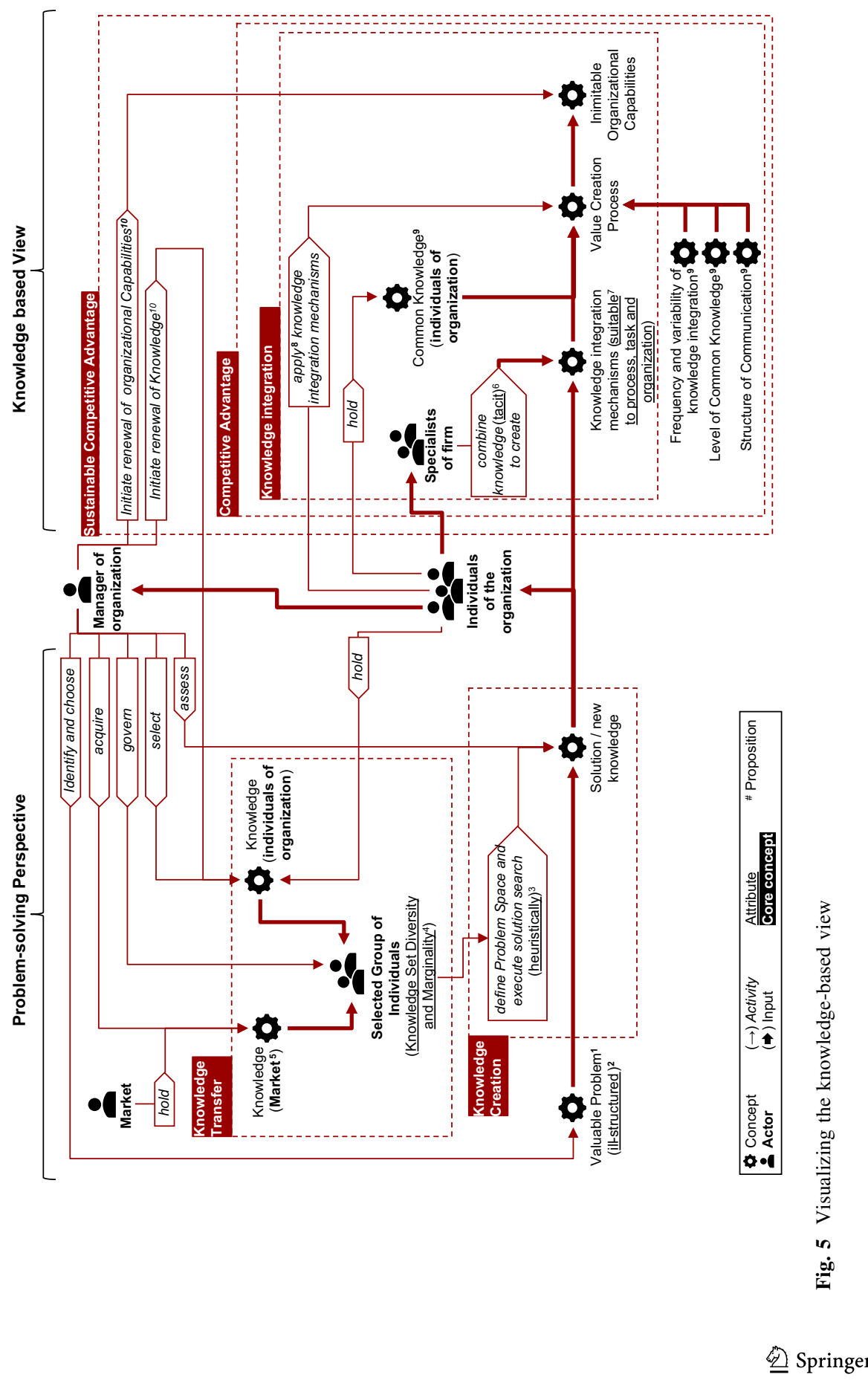




\subsubsection{The process of Analytics initiatives}

Holsapple et al. $(2014,134)$ provide a meta-definition for Analytics, which is "concerned with evidence-based problem recognition and solving that happen within the context of business situations". Hence, solving a problem is at the core of Analytics initiatives and should be the starting point of an Analytics initiative, since it is a requirement for creating usable and valuable solutions, and it is a manager's task to identify this problem (Bose 2009; Barton and Court 2012; Marchand and Peppard 2013). More specifically, "problem" can also be labeled business objective (Viaene and Van den Bunder 2011; Seddon et al. 2017), business question (Bose 2009; Larson and Chang 2016), or an opportunity to exploit (Barton and Court 2012). Absence of this predefined purpose is indicated to result in a waste of resources, creating skepticism toward Analytics rather than data-driven improvement (Lavalle et al. 2011).

Thus, Analytics is endorsed for a focus on handling modern complexity such as dynamic interrelationships and increasing complexity of market and organizational activities and for challenging established business practices, with extraordinary requirements for precision, accuracy, and speed (Kiron et al. 2012; Marchand and Peppard 2013; Holsapple et al. 2014; Kiron et al. 2014). Further, complexity originates from extensive information and data flows (Bose 2009; Beer 2018) or uncertainty (Viaene and Van den Bunder 2011). This requires technically complicated analytical methods with uncertain suitability to the problem and the involvement of various, interacting specialists (Davenport and Harris 2007; Viaene and Van den Bunder 2011; Carillo 2017).

The process for solving these complex problems is described as exploratory and experimental. Based on initial planning, the process consists of well-designed experiments and iterative learning, changing the course of actions due to insights gained (Bose 2009; Viaene and Van den Bunder 2011; Marchand and Peppard 2013; Larson and Chang 2016; Carillo 2017). Hence, this process is very similar to scientific rigor, with experiments being designed with extensive time invested in observing and theorizing unlike IT initiatives (Viaene and Van den Bunder 2011; Marchand and Peppard 2013). Thus, several approaches may be tested against one another to determine the best method for the best solution (Liberatore and Luo 2010; Viaene and Van den Bunder 2011).

Several processes for Analytics initiatives have been described, but they usually show remarkable similarities (Franks 2014). After identifying and understanding the problem, specialists collect, prepare, and explore the data to eventually analyze it and create models to capture the patterns in it (Provost and Fawcett 2013; Franks 2014; Leventhal 2015; Janssen et al. 2017). The results will be presented for evaluation, at least to the intended users (decision makers) and deployment into the business process concludes the process. Finally, the initiative shifts to feedbackbased adjustments and maintenance to sustain the ongoing decision support activity (Larson and Chang 2016).

The results - insight and knowledge — of an Analytics initiative are distinguished according to: (1) discoveries, which provide value in learning, or (2) Analytics products, which provide value in use (Viaene and Van den Bunder 2011; Larson and 


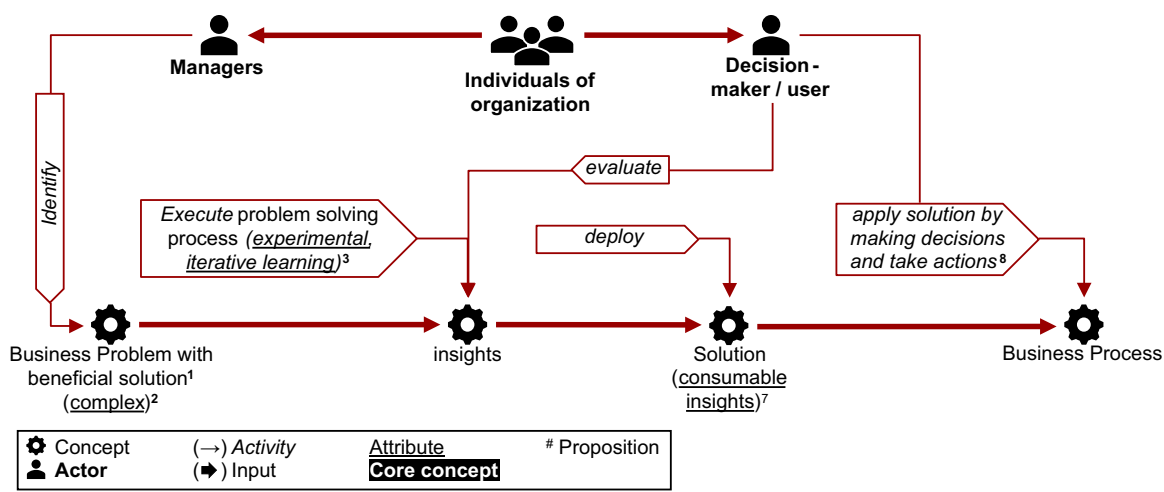

Fig. 6 The process of Analytics initiatives illustrated

Chang 2016). In deployment, these are distinguished by the use of a PowerPoint slide deck or code to deploy models or algorithms (Cady 2017). In both forms, the results are provided to users in a processed way, which is intended to trigger decisions and actions (Davenport and Harris 2007; Bose 2009; Seddon et al. 2017). Consequently, either by taking decisions and actions directly from discovery or by automated rules and decision making/support, the users are benefitting from the effort to transform the Analytics results into consumable insights (Davenport and Harris 2007; Ross et al. 2013). The tasks of Analytics initiatives are illustrated in Fig. 6. Again, the numbers in superscript refer to the propositions explained below.

\subsubsection{Process-accompanying conditions}

Core to Analytics are data, which hold value and knowledge in form of insights. However, insights such as hidden relationships or uncovered patterns are costly to achieve (Bose 2009; Marchand and Peppard 2013; Watson 2014). Moreover, insights must be deduced from the results of analytical methods by interpretation and sense making (Seddon et al. 2017), and subsequently effortfully translated into actionable and understandable decision support for users (Bose 2009; Viaene and Van den Bunder 2011; Barton and Court 2012). This effort is reflected in the literature by descriptions such as extraction, transformation, or unlocking (Wixom et al. 2013; Acito and Khatri 2014; Larson and Chang 2016; Beer 2018). In addition, several data sources with big data characteristics might be integrated, increasing the effort (Bose 2009; Chen et al. 2012; Kiron et al. 2012).

Concerning the specialists executing Analytics initiatives, the jack-of-all-trades analyst idea is rejected for any larger and more complex initiatives (Davenport et al. 2001; Davenport 2013; Carillo 2017). Instead, these rely on cross-functional teams, a mix of technical and business knowledge, with individual team members contributing to insight generation through their knowledge and fostering learning by interacting with other team members (Marchand and Peppard 2013; Larson and Chang 2016; Seddon et al. 2017). While analytical and technical experts carry out the analytical work and ensure technical deployment requirements are met 
(Liberatore and Luo 2010; Larson and Chang 2016), cognitive experts contribute knowledge about how decisions are made and, as a result, how insights are delivered to the consumers of insights (Marchand and Peppard 2013). Further, domain experts prioritize and direct opportunities and inquiries, identify challenges, validate results, and make sure deliverables meet business requirements (McAfee and Brynjolfsson 2012; Wixom et al. 2013; Larson and Chang 2016).

Organizations may also acquire expertise from the market by hiring experts or contracting to external organizations. Organizations that do so may lack experience in Analytics and want to evaluate opportunities (Bose 2009). They may need a broad range of expertise and do not want to invest in building this full range, especially if very specific expertise is needed (Kiron et al. 2012; Carillo 2017). Further, the required expertise may be cutting edge and innovative, and therefore not broadly mastered, resulting in it only being accessible through the market (Barton and Court 2012; Wixom et al. 2013). The process and its associated conditions are illustrated in Fig. 7.

\subsubsection{Requirements and condition for gaining advantages from Analytics}

By itself, the possession of Analytics capabilities or application of analytical methods has no inherent value (Holsapple et al. 2014; Gandomi and Haider 2015; Larson and Chang 2016; Seddon et al. 2017). The value is generated from using the capabilities and created insights, which requires them to be accessible or integrated into business processes to enable insight-driven decisions and actions (Bose 2009; Lavalle et al. 2011; Kiron et al. 2012; Seddon et al. 2017). Hence, the advantages from an Analytics initiative are not provided by producing analytical results but from consuming them for decisions and actions (Ransbotham et al. 2015). This indicates that the responsibility belongs to the users, as opposed to Analytics experts. Thus, the outcomes from executing Analytics initiatives are the users'

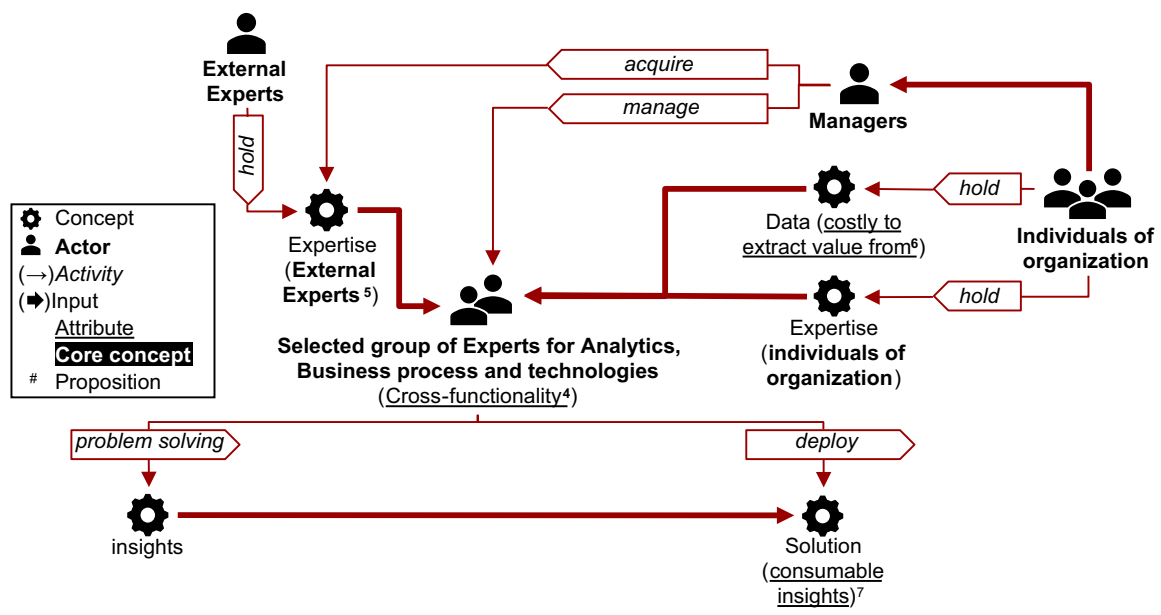

Fig. 7 Process accompanying conditions illustrated 
process and behavior changes and actions triggered by insight-driven decisions (Davenport et al. 2001). If decisions are made but not followed by actions, potential value is missed.

Gaining advantage from Analytics is further facilitated by a data-driven culture. This culture has been presented as strong moderator that positively influences the value generated by Analytics and the absence of which can negate the benefits from Analytics (Barton and Court 2012; Ross et al. 2013; Ghasemaghaei et al. 2017). A data-driven culture is described as a common organization-wide culture that supports, promotes, and embeds shared Analytics-driven ways of thinking, decision making, and acting and accepts data and information as critical for success (Barton and Court 2012; Kiron et al. 2012; Holsapple et al. 2014). A data-driven culture supports a high degree of use of analytical tools to derive insights organization-wide and demands decision making and even challenges prior beliefs based on the insight, which requires analytical skills and literacy (Barton and Court 2012; Kiron et al. 2012; Marchand and Peppard 2013). Insights are proposed to be generated and used at high frequency such as using insights in daily operations, making insights easy accessible on mobile devices, and continuously coaching staff to shift to datadriven decision making (Barton and Court 2012; Kiron et al. 2012; Ross et al. 2013; Wixom et al. 2013). To achieve the aspired-to degree and frequency, organizational structures are established in the form of strategies, policies, processes, and standards (especially for data) supporting the use of analytical tools, communication of analytical needs, and the use of the generated insight (Davenport et al. 2001; Ross et al. 2013; Cao et al. 2015).

The value gained from solutions resulting from Analytics initiatives is not durable. To ensure long-term value, Analytics solutions must be maintained and newly created. For maintenance, user feedback and business outcome have to be analyzed and evaluated to stabilize, adjust, and improve the solution (Liberatore and Luo 2010; Lavalle et al. 2011; Larson and Chang 2016). Further, the solutions can

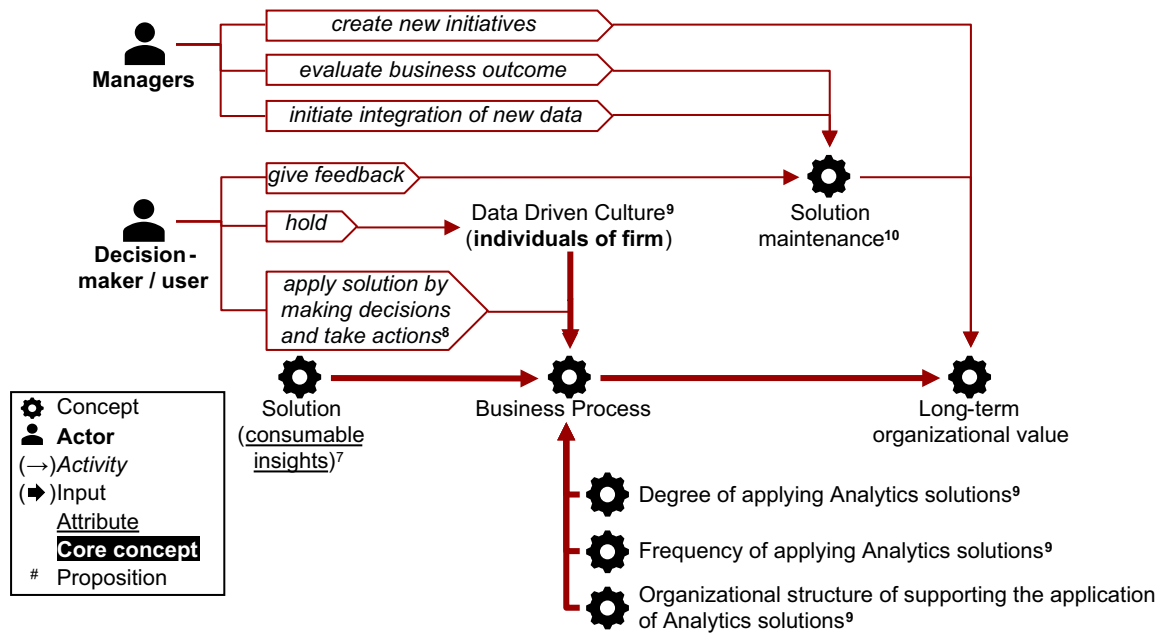

Fig. 8 Enabling advantages from Analytics illustrated 
become outdated or misaligned, especially due to changes in data or the deployment environment, demanding realignment and adjustment to changes and new data (Ross et al. 2013; Larson and Chang 2016; Beer 2018). In contrast to maintenance, creating new initiatives leads to the introduction of completely new solutions or already beneficial solutions to new organizational functions (Seddon et al. 2017). The former is necessary due to the limited lifespan of solutions which cannot be adapted or adjusted (Larson and Chang 2016; Ghasemaghaei et al. 2017). The latter is necessary to broaden the range of functionalities supported by Analytics based on new needs and the intent to distribute the value from Analytics broadly across the organization (Davenport and Harris 2007; Lavalle et al. 2011; Beer 2018). These requirements to sustain the advantages from Analytics are illustrated in Fig. 8.

Finally, direct attribution of value and benefits from Analytics is usually challenging (Larson and Chang 2016). Direct effects are more effective, more informed, and faster decisions (Bose 2009; Cao et al. 2015; Ghasemaghaei et al. 2017), which result in indirect effects that are only loosely associated with the Analytics solution (e.g., improved decision outcomes, improved performance, production of knowledge) (Bose 2009; Barton and Court 2012; Marchand and Peppard 2013; Holsapple et al. 2014). The process of Analytics initiatives creating advantages for organizations is illustrated in Fig. 9.

\subsection{Parallelism of knowledge-based view and Analytics}

The discussion above has presented KBV's theoretical argumentation for competitive advantage generated from integrating and applying knowledge and the practical Analytics processes for generating advantages from analyzing data and applying the results. Both demonstrate immense similarity, assuming data to represent one of several portions of an organization's knowledge. To answer the research question, Analytics is now examined for the rationale behind the practices. This rationale is compared for its resemblance to the argumentation of the KBV. In this research, confirming this resemblance is argued to provide support for an explanation of the competitive advantage generated from Analytics. If the empirical evidence does not show resemblance, either between the practices or the rationale for those practices, the explanation of generating competitive advantage from Analytics based on the KBV is not supported.

Consequently, components of the KBV have been selected and inquiries for empirical evidence have been made for practices in Analytics with respect to those components, including their rationale. The selected components represent key points of the argumentation for generating competitive advantage, which can provide proposed explanations-propositions-for practices in Analytics due to the resemblance between Analytics and the KBV. While the theoretical background presents practices in Analytics to provide evidence for their noticeable resemblance to the KBV and to formulate propositions, they will be treated as unknowns in the data collection. Data collection will also include collecting empirical evidence to explain different practices based on different intentions to disconfirm the KBVAnalytics resemblance, if in fact it does not exist. 


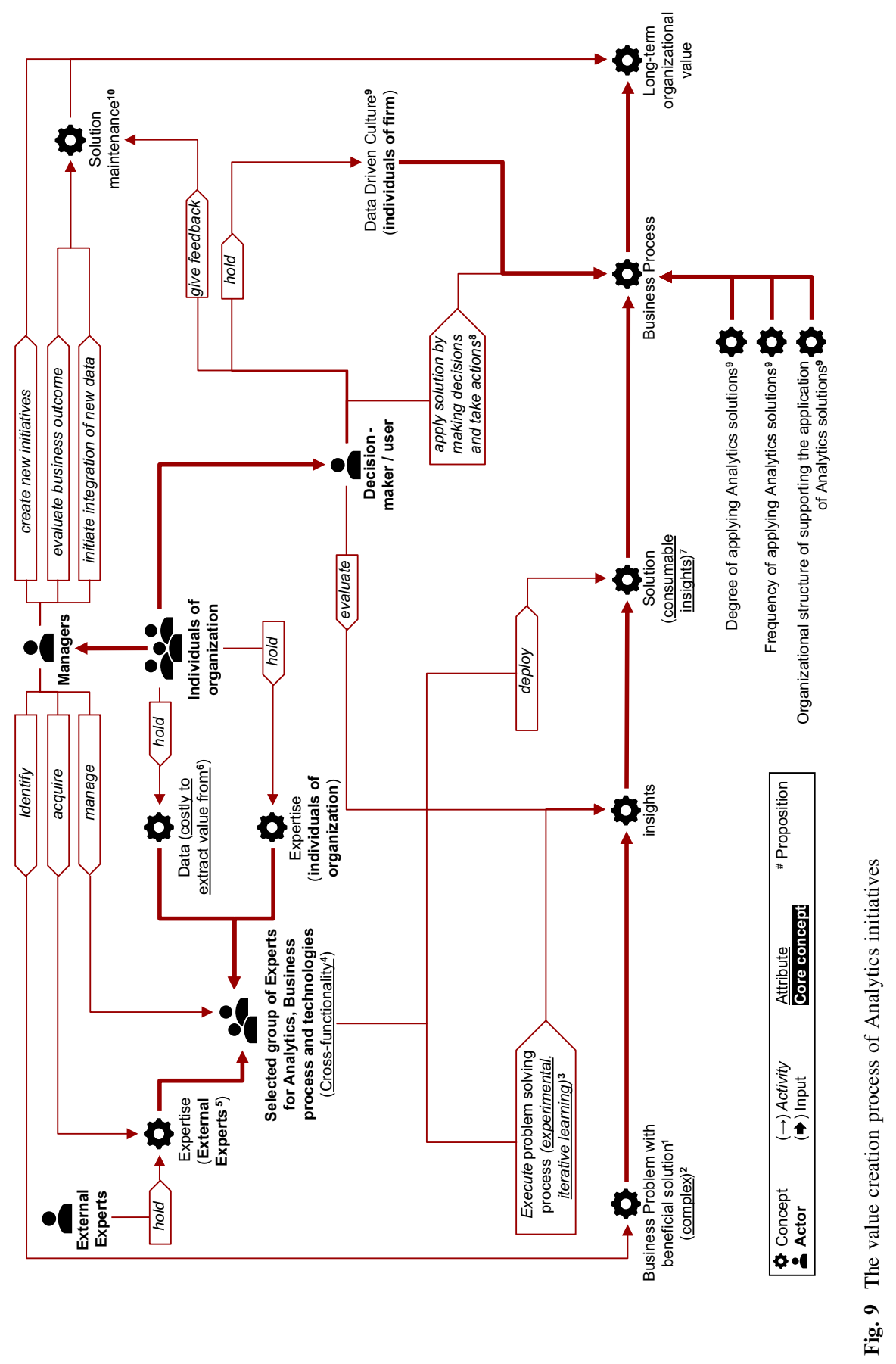


The first selected component is the starting position, which consists of valuable problems to solve for knowledge creation in the KBV. These resemble the Analytics starting point of business problems to solve:

1. Proposition 1: the most promising start for an Analytics initiative is supposed to be a problem (business question, business problem, opportunity to exploit, business target).

Second, the proposed foci of Analytics initiatives are complex issues with unprecedented requirements, which resemble potentially valuable ill-structured problems with high knowledge set interaction. Unprecedented expresses the characteristic of being unsolved for ill-structured problems:

2. Proposition 2: Analytics initiatives intended to create highly valuable solutions are focused on complex issues with unprecedented requirements.

Third, the KBV suggests the heuristics solution search approach for ill-structured problems, resembling the scientific approach of iterative learning in Analytics:

3. Proposition 3: the problem-solving process is an iterative learning process with experiments/tests of solutions giving direction for the next solution iteration.

The fourth component is the knowledge set diversity endorsed in the KBV, which expands the range of solutions, enabled by diverse individuals holding knowledge and taking different roles or specializations. It resembles the cross-functionality of teams contributing to data analysis:

4. Proposition 4: diverse roles in an initiative are relevant and covered by a selected cross-functional team with various different perspectives, expertise, and knowledge.

Fifth, even though it is a controversial aspect in the KBV, inclusion of market actors is selected. Including them for problems with fewer needs of knowledge sharing and access to new knowledge sets resemble acquiring external expertise from the market already available as specialized expertise or gaining access to innovative expertise in Analytics:

5. Proposition 5: external expertise is necessary to exploit market-available specialized expertise or gain access to innovative technologies or methods.

The sixth component selected represents the assumption, as stated above, that data present a portion of organizations' knowledge. The KBV argues that it is necessary to exploit tacit knowledge since tacit knowledge fulfills the characteristics of a resource necessary for competitive advantage, but which is costly to integrate. This resembles the costliness of extracting insights from data:

6. Proposition 6: creating valuable insights and Analytics solutions based on data is costly, with several barriers to overcome.

Seventh, to make use of tacit knowledge, the KBV explains the need for integration mechanisms, which are proposed to integrate knowledge into the value creation process suitable to the task, process, and organization. This resembles the 
creation of consumable insights for users in the form of discoveries and Analytics products in Analytics:

7 Proposition 7: for deployment, the user is provided with consumable insight (one-time insight or an Analytics product) enabling him to work directly with the insight.

The previous component is the prerequisite to allow the eighth component needed for competitive advantage explained in the KBV, the necessity of applying the integrated knowledge in value creation. Resemblance can be found in the user's responsibility to adjust the process and make decisions and take actions from insights explained in the Analytics literature:

8. Proposition 8: after deployment, the user's responsibility is to integrate insight into processes, make decisions, and take actions to create the aspired-to value.

The ninth selected component comprises the moderators for the competitive advantage from the application, which are the common knowledge and the characteristics of its contribution to the knowledge integrated to facilitate competitive advantage. These resemble the data-driven culture on which advantages from Analytics depend:

9. Proposition 9: to enhance the value from Analytics, a data-driven culture has to be built, which is facilitated in Analytics ' broad and frequent use and supporting organizational structures.

Tenth and finally, to make the competitive advantage sustainable, the organizational capabilities from knowledge integration, including knowledge concerning existing capabilities, need constant renewal, which resembles the maintenance of Analytics solutions and their renewed creation to ensure long-term advantages from Analytics:

10. Proposition 10: to maintain value generated by insights and solutions in the long term, maintenance processes based on added data, evaluation of business outcomes, and user feedback are necessary.

The proposed resemblance has two implications. First, if a process to exploit organizational resources exists that leads to advantages and benefits, is employed by organizations, and markedly resembles the knowledge-based view, it would contribute to the validation of the knowledge-based view. Second, if this remarkable resemblance is confirmed and as a result the Analytics process fits the KBV, the advantage from the process would be competitive advantage.

\section{Methodology}

This research aspires to explain how competitive advantage is generated from Analytics. The foundation for that explanation was laid in the theoretical background and the resulting propositions are subsequently tested using empirical evidence. For "How?" questions, the methodology of case studies is appropriate (Yin 2014). Case 
study research has been used to confirm the link between scientific theories and phenomena by scholars in information technology and strategic management. For example, business practices and investments have been linked to the resource-based view and the extended resource-based view to argue for competitive advantage as a benefit of these models (Lewis et al. 2010). A combination of contingency theory, dynamic capabilities theory, and task/technology fit was used to provide a theoretical basis for the field of business process management (Trkman 2010), and the resourcebased view and the related concept of resource orchestration has been used to explore how manufacturers adopt e-commerce (Cui and Pan 2015). Seuring (2008) has underlined the benefits and demand for empirically based case study research to enhance understanding of supply chain management.

\subsection{Research design}

Case study research can be deployed in a way that is exploratory and/or explanatory (Yin 2014), and this study aspires to the latter. Hence, prior to data collection, literature was studied to develop a conceptional framework and testable propositions (Voss et al. 2002; Miles et al. 2014; Yin 2014). The conceptional frameworks are presented in Fig. 5 and Fig. 9. The propositions are presented in Sect. 2.3. The first framework comprises key components of the KBV, which provide a theoretical explanation for sustainable competitive advantage generated from knowledge. The second framework comprises recommended practices for Analytics initiatives that the literature has established as relevant for generating benefits and value, but with limited rationale for their relevance. Using the first framework as reference, case study research is employed to provide empirical evidence to confirm the sustainable competitive advantage generated by Analytics by explaining causal relationships thoroughly. This design fits the case study research objective of providing a causal diagnostic pursued with a pathway strategy that is expected to identify the mechanisms explaining the relationship under investigation and show its plausibility (Gerring and Cojocaru 2016).

For the causal diagnostic pathway strategy, a multiple-case study approach is recommended, preferably with stable background factors (Gerring and Cojocaru 2016). This stable background is implemented by constraining the study to the field of LSCM. This field is exceedingly familiar to the scholars conducting the research. Due to several globally dispersed actors in the varying flows of materials, information, and funds, this field experiences a high level of complexity and process heterogeneity (Simchi-Levi et al. 2003; Bowersox et al. 2007; Wang et al. 2014), which entails potentially interesting cases for Analytics solutions employed for controlling the flows. In accordance, LSCM is considered as an early adopter of Analytics (Davenport 2009) and is considered as a data-rich field with promising returns from Analytics (Kiron et al. 2012; Jeske et al. 2013). Despite these circumstances, many organizations in LSCM show reluctance towards Analytics adoption (Thieullent et al. 2016; Kersten et al. 2017; Brinch et al. 2018), although this audience might develop greater interest based on research insights into Analytics associated with their own field, and may eventually be persuaded regarding the value and benefits. 
Selecting cases from any field regarding Analytics initiatives brings foreseeable issues of limited availability and willingness of experts to respond to research inquiries. Relevant and identifiable experts for data collection are rare and in high demand. Further, with Analytics expected to create advantages over the competition, it cannot be expected that extensive internal documentation will be provided. In summary, it is foreseeable that limited depth can be achieved per case. However, critical analysis of case study research has demonstrated acceptance for limited depth in multi-case research, since multiple cases supporting the results increase the confidence and robustness of results, and provide more sophisticated descriptions and more powerful explanations (Voss et al. 2002; Miles et al. 2014; Yin 2014). As a result, a multiple case study was seen as appropriate to counter accessibility issues while adhering to constraints of time and resources. Further, to increase accessibility, the cultural and language distance to this study was kept short by limiting the sample to organizations operating in Germany. A final number of eight cases were collected. These cases were theoretically sampled such that they were chosen explicitly—not randomly — to achieve greater insights (Eisenhardt 1989).

\subsection{Data collection}

Following established instructions (Voss et al. 2002; Yin 2014), a case protocol was created, including research design, case selection criteria, relevant sources of evidence, and interview questions. The protocol was reviewed by two scholars and adapted accordingly. The sources of evidence to be collected included semistructured interviews, relevant presentations by interviewees and case organizations, relevant publicly available documents from case organizations on LinkedIn, XING, organizations' websites, as well as blog entries, videos, and white papers, in addition to third party reports and articles about the case organizations on relevant topics. These sources of evidence were chosen for triangulation (Yin 2014). The questions were designed to openly ask about aspects of Analytics initiatives, with implied unknowingness, to encourage interviewees to provide detailed explanations and reasoning (Yin 2014). Further, inspired by descriptions on rival explanations/ alternative theories (Voss et al. 2002; Yin 2014), rival explanations about the generation of value from Analytics were developed prior to the data collection and reviewed by scholars, showing a high degree of disjunction among the propositions. The data collection was expanded to include these rival theories. Thus, after answering the open questions about the process aspects in the semi-structured interviews, the interviewees were asked about other explanations (rivals or propositions, depending on the open answer) to obtain their comments on all explanations. Typically, interviewees argued strongly against the rival explanations, enriching the evidence for the propositions but also revealing the incompleteness of the propositions, thus confirming the value of this research design.

The case protocol was revised during data collection to add as much depth as possible by eliminating weaknesses and blind spots (Eisenhardt 1989; Yin 2014), conducting pilot cases for each category, and subsequently reviewing and revising questions and rival explanations. The propositions and their final rivals are presented in Table 1. Pilot studies were conducted for the following categories: (1) 


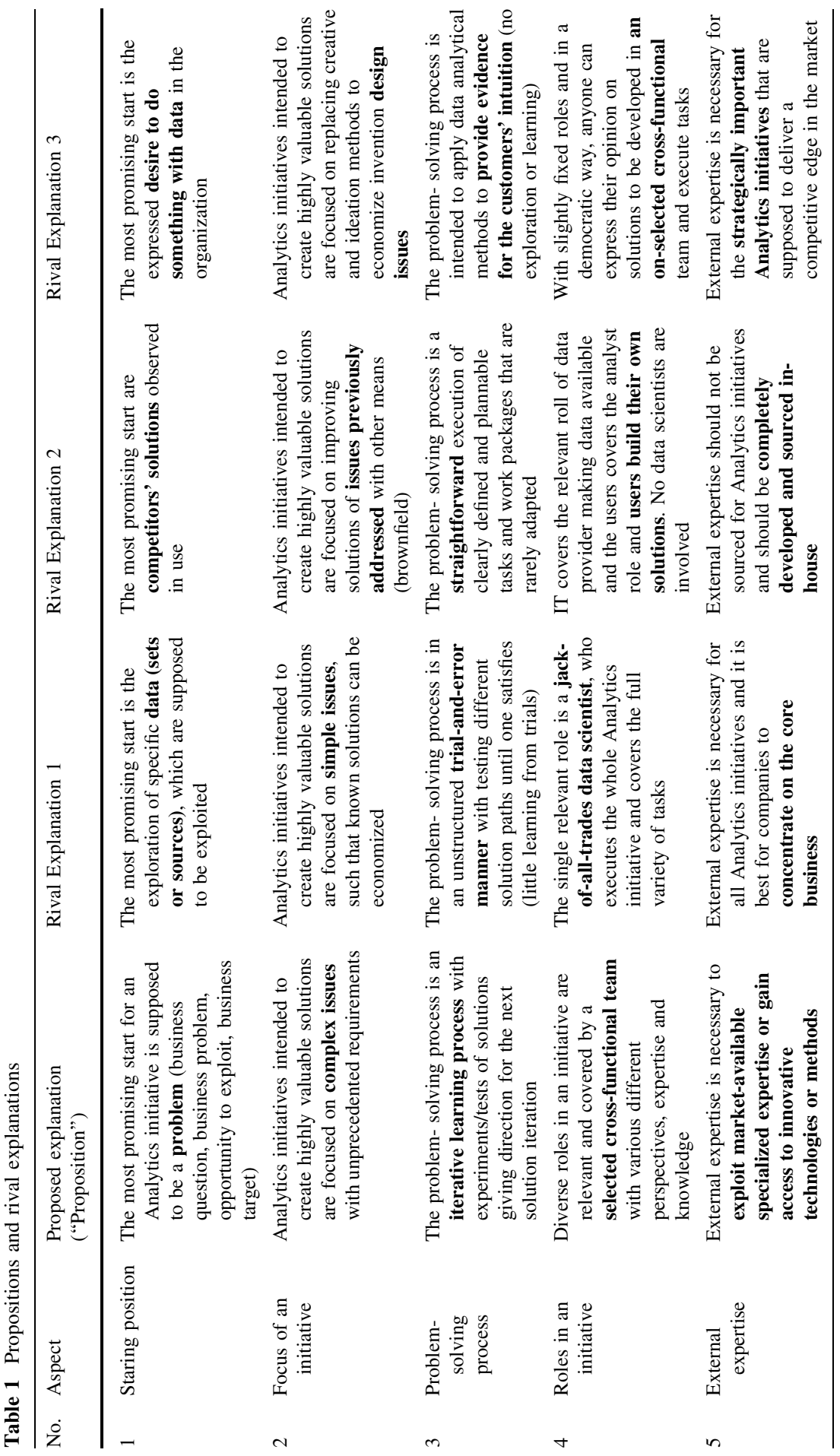




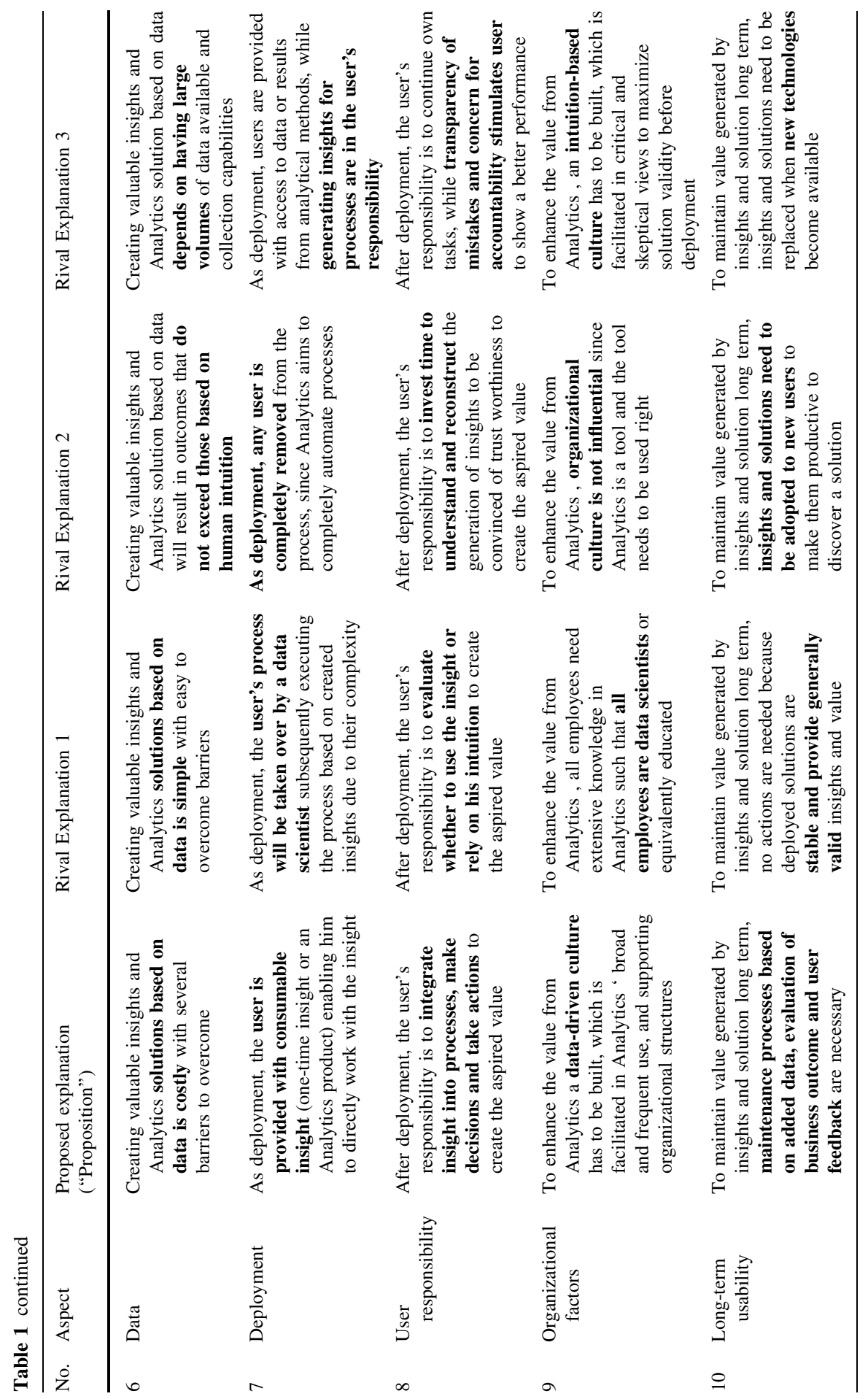


Table 2 Case study interviewees and organizations

\begin{tabular}{|c|c|c|c|c|}
\hline ID & Position & Organization sector & $\begin{array}{l}\text { Organization } \\
\text { size }\end{array}$ & $\begin{array}{l}\text { Experience in Analytics } \\
\text { [years] }\end{array}$ \\
\hline A & Head of Analytics & Software & Small & $11-15$ \\
\hline B & Director Analytics & Software & Large & $11-15$ \\
\hline $\mathrm{C}$ & $\begin{array}{l}\text { Senior manager } \\
\text { Analytics }\end{array}$ & Chemicals & Large & $1-5$ \\
\hline $\mathrm{D}$ & $\begin{array}{l}\text { Senior manager } \\
\text { Analytics }\end{array}$ & Software & Large & $6-10$ \\
\hline $\mathrm{E}$ & Head of Analytics & Logistics & Large & $1-5$ \\
\hline $\mathrm{F}$ & Senior data scientist & $\begin{array}{c}\text { Commercial } \\
\text { vehicles }\end{array}$ & Large & $6-10$ \\
\hline G & $\begin{array}{l}\text { Senior manager } \\
\text { Analytics }\end{array}$ & Pharmaceutics & Large & $1-5$ \\
\hline $\mathrm{H}$ & $\begin{array}{c}\text { Senior manager } \\
\text { Analytics }\end{array}$ & Software & Large & $16-20$ \\
\hline
\end{tabular}

manufacturers using Analytics for LSCM processes or logistics service providers using Analytics ("LSCM organizations"), and (2) Analytics service providers with distinct experience working with and providing services for LSCM. Multiple inquiries to target retail organization did not produce a pilot case, leading to the exclusion of this category.

For the interviews, a short protocol was created and sent to previously contacted experts to evaluate their eligibility for the study and as preparation. The evaluation led to the exclusion of interested but ineligible experts. During the pilot cases, neither snowballing via the interviewees nor direct contacts produced further interviewees. For reasons of consistency, for additional cases also, only one participant in a key position was interviewed per case organization. In summary, eight interviews were conducted via phone and web conference software with a duration of $58.5 \mathrm{~min}$ on average. The interviewees and their organizations are listed in Table 2. Further, a total of 235 documents were reviewed. All sources of evidence were organized in a case database (Yin 2014).

\subsection{Data analysis}

For data analysis, each case is considered as single experiment that is replicated to strengthen confidence in the propositions or provide disconfirming results to shape the theory (Eisenhardt 1989). This was applied to all explanations, propositions, and rival propositions. Thus, as opposed to grounded theory (Strauss and Corbin 1998), the investigation did not synthesize the results from multiple cases, but every case was investigated individually for conformance to the explanations by comparing the evidence to the explanations (Voss et al. 2002; Miles et al. 2014; Yin 2014). Disregarding the rare occasions of no evidence collected for an explanation, which occurred for few rival explanations, the conformance of single explanations over all 
cases was determined by considering the individual results of each case for a single explanation together.

In detail, interviews and documents were analyzed using hypothesis coding (Miles et al. 2014) based on propositions and rivals, since a comparison of theoretical explanations (or predictions) to the collected evidence is recommended (Rosenbaum 2002; Yin 2014). Further, for within-case analysis, the coded evidence for specific explanations was analyzed for each individual case and a synthesized description collected in a matrix case display (Eisenhardt 1989; Voss et al. 2002; Miles et al. 2014). After concluding the within-case analysis for all cases, a crosscase analysis was performed explanation by explanation (Eisenhardt 1989; Voss et al. 2002). The cross-case analysis took place in two steps. First, explanations (propositions and rivals) of each case category (LSCM organizations and providers) were compared within the categories and a description coherent for the categories for each explanation was developed. Second, the descriptions of the categories were compared to determine differences between the groups and to develop a coherent description for each explanation, valid according to the collected evidence.

\subsection{Trustworthiness}

For trustworthiness, the quality criteria of Yin (2014) were considered. For reliability, a case study protocol was developed and discussed with scholars. Further, a case study database was established ordering collected data by case, systemizing individual documents in the associated cases, and recording metadata. Internal validity was ensured during preparation of the research design by documenting and displaying explanation building for the proposition (Sect. 2). During data collection and analysis, internal validity was ensured by explicitly designing rival explanations, which were discussed with scholars, included in data collection, and compared to the propositions in the data analysis (Sect. 4). For external validity, literal replication logic was strengthened by selecting eight case organizations executing Analytics initiatives in LSCM. Construct validity was addressed using multiple sources of evidence including interviews and a variety of documents, as well as by providing the results of this research to the interviewees with a request for comments.

\section{Results and discussion}

This section presents and discusses the results of the case studies regarding support, rejection, and supplementation of propositions. Eight out of ten propositions were supported with consistent results from the case studies, but were supplemented by adjustments and exceptions. The advantage of the research design is to understand how these adjustments and exceptions help to shape the theory that formed the initial propositions. Rival explanations contributed to improved understanding. Resultingly, this section represents the explanation of generating competitive advantage from Analytics. Due to the underlying evidence, this explanation is 
foremost limited to LSCM. However, it is reasonable to assume that the explanation is valid for other domains as well.

In the following, "user" describes the employee in a business process intended to use the results of an Analytics initiative continuously in the form of an Analytics product or an accessible static discovery. "User" further describes an eventual customer of an organization using the results, or an employee whose individual task is automated.

\subsection{Starting position for Analytics initiatives}

According to the evidence, the most promising starting position of an Analytics initiative is a business problem or business question-preferably defined by business users. Interviewees were confident in identifying problems by talking to users in business processes and, if a problem was not formulated by the users, interviewees stressed to identify users and get them involved. Such a business problem allows specification of the proposed solutions. In this way, it becomes more likely that the initiative will develop solutions that users are willing to use, that fit to users' and business processes' needs and requirements, and that typically show better performance. These solutions become more likely to be operationalized and realize their expected value. Their benefits become tangible, and the solutions' impact and value assessible. Hence, initiatives can be prioritized and, since benefits are easier to communicate and explain, create buy-in from sponsors in the form of resources, funding, personnel, and power to overcome barriers. Activities increase in solution orientation and enhance the process (e.g., reduced planning effort; clearer selection of solution-progressing tasks and increased focus of tasks; easier identification of needed resources, data and relevant stakeholders; less delays, determinability of sufficient solution performance levels and test criteria; increased agreement between and more solution-oriented ideas from team members). In conclusion, this approach directs Analytics efforts to create value effectively and efficiently, by considering the value of solutions and costs of developing solutions, while enhancing value realization and reducing overall costs. This resembles the approach of solving valuable problems within the KBV and supports Proposition 1.

However, Proposition 1 is supported with modifications. First, data must be cataloged and inventoried to understand availability, conditions, and developable solutions. Second, Analytics must be the most promising way to address the problem as compared to other techniques.

Rival Explanation 1 to Proposition 1 -starting from data-was rejected. This approach was suggested in some cases as a starting position that is sometimes taken or considered as an option, but was explained to take longer, be likely to result in unusable or irrelevant solutions, and not to motivate sponsors. However, data exploration was declared to be an essential step after problems are understood to create understanding about the data (formats; granularity; timeliness; quality; ability to integrate; what data describes; what data is, is not, and should be collected). Moreover, exploration for taking inventory and cataloging data and data sources is necessary before Analytics initiatives are executed to enable the selection of the most promising Analytics initiatives. This is important, since these first initiatives 
can either build momentum or "burn" the topic, and deficits in the condition of the data foundation soon become apparent. Overcoming these deficits is a time- and resource-consuming endeavor, which neither provides gleaming benefits nor convinces sponsors, but it is an important enabler for executing initiatives.

Starting from a solution seen at competitors, Rival Explanation 2, was rejected as starting point for a competitive advantage. Copying competitors' solutions can be a good start for non-critical solutions. This approach can further be inspiring for organizations reluctant with Analytics. This corresponds to findings indicating an increase of adoption of Analytics if competitors are using it (Lai et al. 2018). However, such solutions are unlikely to have a similar impact at the focal organization. The context dependency and need for adaptation of Analytics solutions is very high. Further, this approach does not put organizations in a position of competitive leadership. For a competitive edge, the observed solution must be made better-an endeavor which is resultingly a business problem.

As it is does not indicate any clear path of action, the desire of doing something with data as advertised in conferences or promoted in press releases, which is the third rival explanation for the most promising start, was also rejected. Any Analytics initiative starting with such a lack of focus leads to discussions about what to do which likely delays any solution and likely creates no valuable solution. However, outside of Analytics initiatives, this desire, formulated and communicated by top management, can push investment decisions, get users to think about business problems that could be addressed with Analytics, and create visibility for Analytics in organizations. Investments are needed for technology, analytical tools, and for developing data collection, storage, structure, quality, and standards. Getting users thinking about long unsolved problems and having their curiosity stimulated is a substantial driver for tackling problems with Analytics. Further, the visibility disseminates benefits and ensures interest in Analytics is sustained.

Regarding LSCM, the case organizations have exploited the proposed starting position to promote the use of Analytics. LSCM users have various problems of transparency and visibility at hand, for example, regarding processes, markets, and competitors. Solutions to these problems provide them with tangible value, including identification of weaknesses, improved planning, reduced cost, faster response times, and reduced firefighting. Addressing these problems created a more supportive approach to introducing Analytics to LSCM.

In summary, Proposition 1 is supported and all rival explanations to it are rejected.

\subsection{Focus of Analytics initiatives}

To gain valuable solutions from Analytics typically means addressing business problems that are complex, because either a lack of appropriate means omitted to solve them so far, or because previously addressed problems remain business critical and enhanced solution performance is desired. Characteristics of complexity are numerous, including time criticality of solutions reaction, need for a high level of transparency across organizations or supply chains, or the amount of data and information to be combined (often exceeding human cognitive capacity). 
Concerning this amount of data, complexity further results from internal or included external factors, actors, object behaviors, and contextual specifics (rules, local characteristics, constraints), which can be interrelated or interacting. Controlling this diversity of input, processed with novel requirements on speed and accuracy, is a salient ability of Analytics and allows organizations to approach completely new problems. The resulting faster decisions and improved decision outputs provide high returns and savings. Further, through maturing in Analytics by executing initiatives, organizations build abilities to address more complex problems. This focus on complex problems for high value and the maturing from solutions resembles the focus on complex ill-structured problems displayed in the KBV. This supports Proposition 2.

Nonetheless, relatively simple problems with high returns-quick winsrepresent exceptions to Proposition 2. Further, the use of Analytics must be reasonable for the problems and should not be excessive, demanding solutions via other means if more appropriate. It was emphasized that initiatives need to be net beneficial.

Rival 1 for Proposition 2, which opposes Proposition 2, was rejected since simple problems, as a focus of Analytics, usually do not provide high returns. In exceptional cases, simple problems may result in valuable insights, and solving complex problems may not provide valuable insights. In this regard, interviewees referred to simple in relative terms, associated with more established methods and tasks, but which still require well-trained experts. Nevertheless, this focus is beneficial in early organizational maturity with Analytics, during which complex problems induce a higher probability of failure and could give Analytics a bad reputation in an organization. Simple problems, in the sense of controllable problems, were described as the foci of initiatives intended to automate decision making with well-understood decision options and whose impact could be comprehensively identified and approved. It was repeatedly explained that even small positive returns are still positive returns, suggesting a cost-benefit perspective as an important paradigm for Analytics.

Rival Explanation 2, of solving previously addressed problems as a focus of Analytics initiatives, was supported as a supplement to Proposition 2. Considering the aspired-to enhanced level of performance for identifying solutions to business problems through the presentation of an ill-structured problem with unrevealed and unmastered knowledge set interactions, this rival explanation resembles the KBV. Implemented solutions to business problems are usually justified by their business criticality. If this criticality remains with a need for enhanced solution performance and Analytics is likely to deliver it, an Analytics initiative consequentially creates high value comparable to completely new problems, as specified in Proposition 2. This value can result from higher performance, higher efficiency, more relevant insights, standardization of solutions for increased usability and accessibility (e.g., standardizing spreadsheet solutions), or new approaches (e.g., from reactive to proactive). Such initiatives support maturing in Analytics, since many aspects of the problem are well understood, benefitting the solution development. Again, it was argued that benefits exceeding the costs and proper priority are deciding factors for executing initiatives. 
The third rival explanation was rejected, since Analytics is not focused on replacing ideation and creative methods. It is rather a supportive input to ideation and guides innovations into the right direction to exploit opportunities. In contrast, creativity is vital to identifying the right business problems to address. Analytics can support the development or even be part of new value-added features, services, products, contract formats, and business models, while creativity and ideas are needed to design their monetization. However, the case studies presented a tendency to address internal process improvements with higher priority compared to building customer facing solutions.

According to Proposition 2, the complex issues currently the focus of Analytics initiatives in LSCM is manifold. LSCM organizations want to understand customers (what they do and need), assess process quality in real time, and predict critical conditions of systems and assets such that countermeasures are resource and cost efficient. Asset fleet utilization is expected to be increased beyond non-Analytics limits and needs to gain capabilities for the same day and same hour delivery under pressure on margins. Finally, more individualized services are anticipated to be provided due to Analytics, which is a paradigm shift from economies of scale and scope.

In summary, Proposition 2 is supported with acknowledged exceptions and Rival 2 is supported, which supplements the proposition. Rivals 2 and 3 are rejected.

\subsection{Problem solving process}

Case studies presented the problem-solving process of Analytics initiatives to be iterative, with future iterations being guided by and extensively dependent on learnings from previous iterations. Further, the solutions of iterations are not put into trial in processes to see whether they fail or not. They are rather considered as intermediate states (proof-of-concept, pilot, prototype), which are the basis for discussions between solution developers and users, joint interpretation of results, validation of results, and making use of users' knowledge to guide the problem solving process. According to interviewees, such iterations simplify the integration of several perspectives. Iterations are further supported by establishing the objective of initiatives at an early point to reduce ambiguity (e.g., memorandum of understanding, letter of content), and by agile sprints, as recommended by Larson and Chang (2016), in which tasks are focused on the next agreed intermediate state. As a result, this approach ensures knowledge transfer in iterations, in the form of user feedback, to ensure selection of the relevant knowledge of the users (addressing intermediate solutions' deficiencies and gaps to expectations, domain knowledge guides solutions' improvement) as well as from the solution developers (methods to implement users' feedback). Thus, intermediate states are used for anticipated necessary interaction between users and developers. This resembles the heuristic solution search presented in the KBV and supports Proposition 3, although constrained by certain adjustments.

Adjustments emerge from the rival explanations. First, while the problem-solving process is iterative, initiatives follow a certain, structured approach, such as the repeatedly mentioned CRISP-DM model. One interviewee explained: "These 
projects are deeply unstructured, but you can approach them very structured". Second, Analytics products are also deployed with the intent to advance the solution based on user feedback, which at first glance resembles trial-and-error. However, this requires a quite advanced solution that is improved from feedback instead of radically changed.

Rival Explanation 1 to Proposition 3 was rejected because creating a valuable Analytics solution that users like to use requires continuous feedback during solution development. A trial-and-error style approach based only on past experience with positive and negative outcomes in developing solutions, neglecting necessary user and context adjustments and attempting to deploy without prior user feedback, is likely to produce inappropriate solutions that are not accepted by users.

As explained above, problem solving is not straightforward, and Rival 2 is rejected. But structures for initiatives exist that guide users through various welldeveloped phases (e.g., CRISP-DM phases) that enfold the problem-solving iterations. Iterations and agile sprints do not facilitate chaos or lack of control. They need rules, structures, and documentation requirements such that the organization can repeat, recreate, and learn from initiatives. In this regard, it has been emphasized in the case studies that Analytics is not "just algorithms", it is the whole process around the algorithm that intends to generate value for organizations. Still, the process may be disrupted by stakeholders changing opinions, unclear decision structures nullifying previous decisions, or unanticipated data and technology issues setting the initiative back. Increasing maturity with problems, technologies, and Analytics reduces these disruptions.

While the problem solving is guided by users' knowledge and intuition-a distinction that will be discussed below-it is not intended to use Analytics to confirm users' intuition or expectations to "play politics". Thus, Rival Explanation 3 is rejected. If the intuition was correct, the generated benefit has more certainty in actions providing limited value. If it is wrong, results that provide some confirmation could be fabricated, but this does not provide value, would not lead to actions in the best interest of the organization, and would be malpractice. However, interviewees also explained they had not observed this behavior. The strength of Analytics is seen in overturning existing business thinking and practices if a better outcome can be achieved differently.

It was emphasized that the iterative approach is specifically needed in LSCM to understand the perspective on a problem. Regarding forecasting of demand and capacity or the assessment of quality and performance, there are various perspectives in LSCM that require different aggregation of timescales, entities, and processes for the seemingly same problem. Iterations are essential to filter the correct perspective and informational needs.

In summary, Proposition 3 is supported, with all rivals being rejected.

\subsection{Roles in Analytics initiatives}

The evidence shows that Analytics initiatives are based on constantly interacting cross-functional teams with members filling different roles to perform different tasks. In particular, the inclusion of users, permanently or accessible at short notice, 
was highlighted. Users' inclusion enables solutions' dedication to users' needs, their operationalization, and their impact on business processes, since users have deep knowledge about the process (understand data and contextual meaning, understand required solution performance, know local specifics of decision making, special business rules, and requirements, and factors impacting the outcome). They help to identify difficult-to-reach data sources, as one interviewee explained: "Excel spreadsheets are very popular, again and again". Apart from users, diversity of team members provides diverse skills and specialized knowledge, and diverse talents, perspectives, experience, and interests (a vital motivational factor). This mix enables more, better, and unconventional innovative ideas in Analytics initiatives, provides synergies, and accelerates the problem-solving process. Tasks can be distributed across the team to gain efficiency from specialization and manage the high workload. This resembles the KBV's argument for a group of specialized individuals required for knowledge creation from combining diverse knowledge sets and supports Proposition 4. Members were also explained to speak different "languages" due to their different cognitive concepts requiring a "translator" who has enough knowledge about the different roles to understand and connect the team members, comparable to the argument in the KBV. This role was explained to be even rarer than great data scientists.

Again, an exception applies to Proposition 4, since different roles may be filled by the same team member depending on the size and complexity of the initiative. Not all Analytics initiatives are intended to place an organization ahead of the competition, change the business model, or master the most complex business problems. Thus, unconventional and innovative ideas, synergies, or management of high workloads are not required for all initiatives.

However, uniting several roles has a limit and as a result the jack-of-all-trades data scientist specified in Rival 1 was rejected. Interviewees explained that their organizations' data scientists usually have a strong mathematical background (mathematics, physics, statistics), often with a $\mathrm{PhD}$, since their tasks and core expertise are to make the most of data (analyzing, modeling, creating algorithms). They agreed that it is easier to learn new data tools with this background than the other way around. However, jack-of-all-trades data scientists were sought to find, but in the rare instances of finding one, they were extensively more expensive than a diverse team, thus eradicating their benefits. Data scientists are generally expensive and should focus on the data analytical tasks they are best trained for, as a matter of resource efficiency. They might do small initiatives on their own, but other initiatives would be physically challenging due to the workload- "It does not scale". This data scientist would rather be a single source of failure and interrupt the entire initiative in case of illness. Moreover, an idea of omnipotent data scientists contradicts the value of business experts and users, who have effortfully acquired knowledge and experience handling complex tasks. This idea disrespects them and their contribution, which can result in losing their eventually needed collaboration.

Rival Explanation 2 of users executing the initiatives was rejected, since users usually lack knowledge and experience needed for Analytics initiatives or are occupied with their business processes, on which they are expected to work. Further, data literacy and knowledge on technologies may also be missing, and training 
would be extensive and require their affinity for the topic. With the so-called "selfserving Analytics", users are increasingly getting involved with analytical tools, but these are often specifically developed or adjusted for users' empowerment (e.g., supporting workflow, providing relevant options as selectable) and require prior Analytics initiatives for their development. Thus, established methods are democratized instead of users working on leading edge problems. However, this reduces the workload of data scientists, who can then work on complex problems and accelerate gaining value from those.

Case studies indicated that the involvement of employees only weakly related to the business problem can be helpful in certain situations that require creativity and additional perspectives. Marchand and Peppard (2013) recommended such action as a strategy to introduce new ways to solve problems and overcome myopic views on data initiatives. However, this could also lead to revisiting already dismissed ideas and more discussions. Any strong voice intentionally or unintentionally putting their own needs first could divert the configuration of the intended solution away from users' needs. Further, input not relevant to the solution can disrupt and delay progress, which creates inertia in initiatives. Thus, Rival Explanation 3 was rejected.

In LSCM, putting the proposed cross-functional teams into practice comes naturally. LSCM as business function or business model usually interacts with many cross-functional actors and must fulfill the role of integrator. In LSCM organizations with an end-to-end vision of their supply chain, this is scaled to cross-organizational teamwork. Thus, working in cross-functional teams is the status quo for LSCM and nothing new due to Analytics.

Summarizing the above, Proposition 4 is supported, and all rivals have been rejected.

\subsection{Including external expertise}

Externals such as providers with high Analytics maturity are included in Analytics initiatives to develop better and cheaper solutions, because they are familiar with the problem and their expertise is an efficiently purchasable commodity. Including externals was further reported as beneficial for gaining access to specialized, innovative, and niche analytical methods and technologies. In accordance with Proposition 5, they are included when organizations have no interest in building expertise in rarely needed methods and technologies. However, organizations also include externals to build expertise and develop self-sufficiency, which goes beyond the proposition. Evidence clearly displays the adoption of Analytics as an effortful maturation, whether initially or for adopting Analytics innovations in mature organizations. This maturation is accelerated and reduced in stress and cost by appropriately knowledgeable externals with the intention of supporting maturation by executing co-creative Analytics initiatives. During maturation, externals can generate "buy-in" from sponsors by showcasing completed initiatives and value from Analytics and their experience reduces the risk of failure and "burning" the topic in-house. However, the goal must be to develop internal expertise and selfsufficiency, since this is vital for the organization to develop inherent ideas for initiatives and trust from users. Thus, these co-creations are successful if externals 
eliminate the need for their expertise (not necessarily for their technologies), while this success provides a basis for new collaborations on different or more advanced topics. This partly resembles the inclusion of market actors for problems with fewer requirements and access to more diverse knowledge as explained in the $\mathrm{KBV}$, but Proposition 5 must be rejected since it does not represent the full situation and misses an essential part of the inclusion of externals into Analytics initiatives.

More diverse but regular modes of cooperating with externals were reported, also not covered by Proposition 5. These include using externals as an "extended workbench" to gain flexibility, or for critical and urgent problems, regardless of the Analytics maturity of the organization. Further, externals may have access that cannot be substituted in other ways, such as solution providers that include data from other customers into a solution to enhance it. A minority of organizations always include externals to gain more diverse perspectives. Also less regular and outside the narrow focus of using externals for knowledge sourcing is the sharing of knowledge and resources to develop a collaborative solution for the market in a strategic partnership.

Rival Explanation 1 of companies focusing on their core competencies and leaving Analytics initiatives to externals was rejected in consideration of competitive advantage. While uncritical business problems with mature solutions exist, saving time and costs, building organizational self-sufficiency in Analytics was emphasized as essential for creating a competitive edge from it. Analytics is anticipated as one core component of future competitiveness as part of product and business models and solely relying on externals limits an organization's capacity to build maturity for this anticipated competitive environment. Further risks mentioned include the lack of the ability to create Analytics-enabled business ideas or loss of these ideas, loss of data and control over data usage, and loss of control over solution quality. Certain Analytics initiatives must be done internally, due to privacy, confidentiality, and data security concerns, or because regulations prohibit data sharing. Thus, scholars' recommendations (Sanders 2016; Lai et al. 2018) to remain in the "comfort zone" of core competencies should be subject to critical consideration and careful assessment.

Since it displays an extreme position intended to broaden the insight generated from case studies, similar to the previous rival explanation, Rival 2 of Proposition 5 was rejected. Completely developing and sourcing Analytics internally limits the organization. Externals can be cost-effective providers for solutions with high maturity for uncritical business problems. Regarding competitive advantage, their support can accelerate the process, reduce costs, reduce risk of failure, and introduce new ideas on problem solving. As a critical side note from interviewees, not all externals can necessarily contribute these benefits, but market leading providers should. Organizations have different core businesses and competencies, and they should not disregard the development of innovative Analytics concepts and technologies happening outside their own organization.

Organizations should have full control over strategic initiatives and customer facing services, Analytics or other, but externals, if bound to confidentiality, can have an important impact on strategic initiatives by introducing additional ideas and information. Thus, Rival Explanation 3 is inconclusive. There was no consensus to 
be found in the case studies. Some strongly rejected the explanation, arguing for the vital importance of strategic initiatives, the risk of losing new revenue streams to cocreators, and different understanding of customer needs. However, other cases appreciated the use of externals for the innovative solutions that could be created in partnerships and acceleration of implementing strategic initiatives leading to first mover advantage.

While the case organizations represent leaders with relevant insight on this research, only a few LSCM organizations build internal Analytics expertise. Many intentionally take follower positions and, thus, source Analytics expertise completely externally. This does not contradict Proposition 5, since it has not been experienced that such organizations gain competitive advantage from their Analytics initiatives. Further, case organizations have even started to push competitors to use Analytics, since they perceive this reluctance as a risk to the field.

In summary, the proposition is rejected, since the proposition only covers parts of the rationale in the data. Rivals 1 and 2 are rejected and Rival 3 is inconclusive.

\subsection{Data as a resource}

Data as a resource was a controversial topic in the case studies. It is the core of Analytics, holding the insights that enable valuable opportunities, but it is also a source of challenges and frustration. Interviewees expressed their frustrations vibrantly: "because every customer says: 'the data is there, it's great, it's no problem at all.' It's not like that, it's never like that." Data issues can impede its use for Analytics, including: (1) integration of data (different formats, timeliness, frequency, granularity, or data definition of business objects, missing context or technical accessibility), (2) data quality (missing annotation, incomplete data, uncertain correctness, errors from sensor failures), or (3) data management (ensuring data security, missing overview over data, unclear responsibilities for data quality and security). Further organizational issues can result in denied access to the resource, including: (4) protectionism (unwillingness to share data, missing trust between supply chain partners) and (5) infrastructure (evolved data silos, differing decision rules for similar data). These issues reduce the usability of the resource and the value of solutions, considering the repeatedly mentioned "garbage in, garbage out" principle. However, they concern handling and care of the resource and become less relevant with increasing maturity in Analytics due to standardization and improvement efforts driven by needs inside and outside of Analytics initiatives. The relevant issues arise from the insights held in the data resource. To generate insights from data to solve a business problem, two steps are necessary. First, data must be analyzed with quantitative methods and algorithms, which may not be able to uncover comprehensive insights about the entity the data was collected about. Second, the results from these methods must be interpreted for their relevance and impact on the business problem, which demands additional knowledge on context and domain that cannot simply be substituted. Thus, the full insight covered in the data might not be transferred to the business problem of an Analytics initiative. Further, data might not be collected for technological reasons, data security reasons, prohibition by law, and due to missing installations of 
sensors. Hence, insights may not be transferable completely with data as the carrier. Additionally, resulting from this and previously discussed evidence, all these activities of collecting, managing, and analyzing data and interpreting results are usually time, resource, and cognition intense, making the transfer of insights to solve a problem with data as carrier costly. This resembles the characteristics of tacit knowledge as explained in the KBV, and supports Proposition 6, again with supplementation.

An emphasized adjustment to Proposition 6 is the progress in methods and technologies, which results in decreasing costliness of extracting insights from the data. Further, in exceptions, costly Analytics cannot promise to extract insights that are valuable, and in other initiatives the extraction of insights is not costly, but the insights are valuable.

The extraction of insights from data being simple, as specified in Rival Explanation 1 of Proposition 6, was rejected. Analytics initiatives with simple to extract valuable insights for problems or organizations become fewer with increasing maturity of organizations in Analytics. Moreover, the process of insight extraction from data was explained to be cognitively effortful and time and resource consuming.

Evidence concerning Rival Explanation 2, which proposes that insight extraction from data does not lead to greater value than insight from human intuition, is inconclusive. The inconclusiveness centers around the ambiguity of the term "intuition", which describes some "gut feeling" or guesswork but also human knowledge, experience, and access to information not represented in analyzable data. The latter consists of cases in which Analytics insights cannot exceed human knowledge, problems cannot be modeled, discoveries are irrelevant, analytical methods miss patterns, or data from different sources are inconsistent, leading to suboptimal decision support. However, there are also cases in which Analytics solutions exceed human knowledge and experience. After all, human knowledge and experience are often necessary to create valuable Analytics solutions in the first place due to understanding of interrelationships, sense making of data, or feedback guiding the problem-solving process. Combining human knowledge and experience with Analytics was reported to achieve the best business outcome, making Analytics dependent on humans' abilities but not declaring human intuition as superior to Analytics.

The dependency on large volumes of data for valuable insights, stated in Rival 3, is also inconclusive. While small data can also create valuable insights, the data volume can be substantial for enhanced insight creation under certain conditions. The additional data volume must explain more aspects of the business problem, either from a greater variety that allows the explanation of more effects, or from more equivalent data but with higher diversity of observations. Resultingly, more precise solutions must be creatable or new questions answerable. Further, the volume must be exploitable (technically manageable, with provision of results before the value of the insights degrades) and have good quality. Most importantly, and sometimes missing from big data discussions, the larger volumes of data only provide value if they fit with the problem. In contrast, if there are privacy or security concerns with the data, more data can result in higher risks. 
Regarding LSCM, a complexity in analyzing data arises from LSCM being process focused resulting in according data creation, while classical IT systems are oriented to the structure of the organizations. Hence, analyzing the process can be challenging. Further, LSCM operates in dispersed locations. Thus, different markets of operations bring different business rules applied to the same data and generate differing insights. In established and grown organizations, the dispersed locations (manufacturing, storage, fulfillment), globally and locally, have been set up with heterogeneous and now outdated systems, which either prohibit data access or provide data that cannot be integrated, making the knowledge technically tacit.

In summary, Proposition 6 is supported, Rival 1 rejected and Rivals 2 and 3 inconclusive.

\subsection{Deploying Analytics solutions}

Evidence emphasizes a deployment of Analytics insight such that decision making is supported in a consumable form, which accelerates the business process, is more relevant and appropriate to the decision, or enables users to consider a wider variety of business questions. The value from Analytics is eventually measured by the resulting business or process performance, since Analytics is supposed to result in better or faster decision making. However, the desired value can be missed if deployment of insights is delayed or leads to wrong decisions because of missing consumability. A lack of consumability prevents users from accepting and using the solution or reduces productivity. Consumability is influenced by a variety of characteristics. The solutions should fit to the process and users by being intuitive for users and intuitive in regard of the process, available when needed, attracting attention for relevant situations (alerts, visualizations), reflecting business logic, and, if needed, allowing the use of preferred devices including mobile technologies. Complex decision making becomes more consumable by reducing the steps to the decision, reducing decision-making effort especially under pressure, and making insights usable without deep analytical knowledge. For uncertain decision making, consumability includes access to additional insights on demand and to the expected consequences of the recommended decision. In summary, solutions must be timely and appropriate to the business impact of supported decisions. Insights on the same problem for different user groups (e.g., maintenance vs. new product development) are consumed differently and require different deployments, underlining the need for involving users in the problem-solving process. This consumability is established by a deployment that is fitted to the process and users to ensure beneficial usage, reflecting the suitability of the knowledge integration mechanisms to the process and organizational characteristics for efficient knowledge integration. This supports Proposition 7, with subsequent adjustments.

Necessary clarifications must be made to adjust Proposition 7. First, consumability does not exclude training. Second, consumability is not the uniquely important aspect, since the deployed solution must also fulfill technical and legal requirements (scalable, secure, legally correct, licenses are paid).

Rival Explanation 1 for Proposition 7 describes the migration of decision making supported by Analytics solutions into the responsibility of data scientists. This is 
rejected. The cases emphasized users must remain in the process and be enabled and empowered by appropriate solutions. The users have the necessary experience and knowledge for the processes, can interpret and identify the most appropriate decisions and actions given the insights, and should remain responsible for decisions and actions. Analytics solutions cannot cover the full decision-making process and tasks of users, which require understanding of the business environment, strong process-related skills for which users are educated, and ideas to improve processes with methods outside the realm of Analytics, which data scientists cannot substitute.

Analytics products are intended to reduce user effort, including the automation of certain repetitive and less complex tasks. However, replacing the users completely, as stated in Rival Explanation 2, is neither desired nor technically possible, leading to rejection of the explanation. The intention is to reduce users' tasks that are time intensive but do not provide much benefit to users or organizations (e.g., manual data collection, manual data integration), while this study did not investigate whether this is in the interest of the user. The automation of decision making requires a proven and established solution with consistently superior performance for the user and a backdoor process for eventual changes. In complex decisionmaking processes, such automation is often technically not possible but instead requires users' abilities such as ingenuity, creativity, and ability to interpret and understand the impact on the process, and to evaluate the best course of action. As also noted by Roßmann et al. (2018), human skills will remain necessary for decision making. Automation cannot transform a business in the way a knowledgeable design decision by a human can do. However, certain jobs are becoming less attractive or require actions beyond human abilities, such that it becomes necessary to develop automated solutions. Further, Analytics solutions may lead to centralized decision making or the ability to scale tasks and replace users in that way. Ironically, interviewees reported the increasing automation of analytical tasks.

As discussed above, users are increasingly enabled through self-service Analytics. This depends on Analytics products developed in Analytics initiatives to create solutions that provide access to relevant data-data democratization-and analytical tools fitted to the user. As Guerra and Borne (2016) described, democratization of data implies easy access based on standardized metadata, access protocols, and discovery mechanisms. It is not practical to leave the generation of insight and solution deployment solely in the hands of the users, who receive the results from some analytical method, rejecting Rival Explanation 3. Self-service or self-sufficient Analytics (for the users) depends on the users' affinity for Analytics, their willingness to learn, and the complexity of the problem. Certain insight generation, self-sufficiently executed by users, contributes to building trust into Analytics solutions such as customization (change reports, build own apps) or further data exploration. This basically efficiently exploits users' knowledge on needed data, understanding of processes, and ability to interpret data and insights in context.

The field of LSCM displays an interesting occurrence regarding this proposition, because automation is a major aspiration, but not to cut jobs, as feared by automation opponents. The intent to automate is stimulated by the lean mindset, a 
central mindset of physical process optimization in LSCM, which focuses on reducing non-value-adding activities. Thus, automation intends to free employees from these, often repetitive, activities and provide more time for value-adding activities and innovating based on their expertise. The value of LSCM experts was strongly emphasized in the case studies and the irreplaceability of the experts' knowledge by automation was expressed repeatedly.

In summary, Proposition 7 is supported, and all rivals are rejected.

\subsection{The responsibilities of the user}

Insights from Analytics solutions must be integrated into business processes to result in decisions and accordingly taken actions. Similarly, discoveries must be operationalized by supporting decisions and (corrective) actions users are responsible for taking to improve business processes or eliminate inefficiencies. This seems rational, but is not always how users act. These decisions and actions from Analytics solutions are necessary to generate return on the investment. Unused solutions may result in additional investments, since the business problems appear to be unsolved. As one interviewee described: "if your weather forecast says rain, but you don't take an umbrella and get wet, you cannot blame the weather forecast nor the umbrella manufacturer". The purpose of Analytics is not analyzing data-as already indicated in the literature (Chae et al. 2014b). The purpose of Analytics is to improve decision-making and business processes through integrated, Analyticsenabled support or automation, provided Analytics is the tool in an organization's toolbox that achieves the desired solution with superior performance or cost efficiency. Further, integration includes process changes by allowing users to collect feedback on the solution's performance, such that transparency of results and longterm improvement are enabled. Since this underlines the importance of applying the insights from Analytics solutions during the value creation process of transforming input to output, Proposition 8 is supported with subsequent adjustments.

As clarifying adjustments, the method of integration and the level of change of the business process strongly depend on the insights, such that discoveries in presentations and reports with longer periods of validity are integrated differently as compared to alert systems. Further, the solution must be created such that integration into the business process is possible, and, in accordance with the results of Srinivasan and Swink (2018), the business processes may have to be stabilized first such that integration is possible. It should not be implied that all new insights are dominant over previous decision making. The solution from an Analytics initiative may just provide additional insights for decision making in the process.

If a deployed solution is established and feedback returned, users should not evaluate whether to use or to ignore the decision support recommendation or assistance because, for example, it does not fit their intuition. Thus, Rival Explanation 1 to Proposition 8 is rejected. However, such an evaluation would be part of the solution's refinement and validation during deployment, which benefits from users' knowledge and experience. Further, decisions themselves are evaluation processes, which are supported by Analytics solutions and usually include further sources of information. For making the decision, users should not blindly follow the 
actions recommended by Analytics solutions, since these could also include distortions (e.g., failing sensors). But risk is no excuse for generally or conveniently ignoring. Again, critical business information might not be covered by available data and models and thus the Analytics solution may be overruled. The critical information available to the user must be sound. Further, there might be additional decision options that are severe and purposefully unavailable in the Analytics solution. However, this argument applies only in certain situations.

Interviewees clearly emphasized that users should not recalculate the results provided by Analytics solutions. Thus, Rival Explanation 2 is rejected. Solutions should usually be consumable, reducing the needed mathematical skills of the users. The effort required to recalculate the results would likely eradicate the intended time benefits and would be scarcely manageable by the user, especially with complex calculations connecting several models. However, change aversion results from lack of understanding. Hence, to build trust, how the solution works and generates the insight should be explained to users. This might include recalculation in some form.

While transparency can reveal mistakes, it should be used as a chance to learn instead of building pressure. Handled as an opportunity for learning and supporting users, Analytics is perceived as beneficial and builds demand. Used to apply pressure, solutions will not be accepted and applied, and data will not be shared willingly. Thus, Rival Explanation 3 is rejected. Transparency should be created while remaining ethically sound and ensuring users' privacy with established data security. Organizational authorities should monitor this, while also clarifying and creating consensus about ethics and privacy, setting up rules to follow and enforcing them ("data governance"), and providing users with understanding about data collected related to them, the reasons for collection, and how to take control of their own data. A rising need for governance was foreseen by Carillo (2017) and his demand for research attention is supported by this research. Increased transparency should be beneficial for organizations and users, and benefits must be communicated to users. Otherwise transparency could become a source of fear (blaming, loss of power, job automation). However, limitations should be reasonable and not overprotective or generally refuse transparency. Transparency can be achieved without any relation to users, and data on users can be separated from data regarding the subject of analysis. Making organizations transparent is needed for business success, safety, and risk management, and to identify intentional wrongdoing.

In LSCM, the responsibilities described in Proposition 8 result directly from the business problems tackled. The aspired transparency on changing conditions does not affect the situation for the organization. The situation is changed when users take decisions and actions accordingly, such as redistributing resources, rescheduling, or reordering. Faster response and broader insights on the changed conditions with their consequences should increase available decision options with resultingly better outcome for the organization. However, evaluating the options, taking decisions, and triggering actions are the user's responsibility.

In summary, Proposition 8 is supported, and all rivals are rejected. 


\subsection{Organizational factors of Analytics initiatives}

Several organizational factors are identified in the evidence, which enhance the value generated from Analytics. However, while these are related to a culture of open and positive interaction with data, this culture was neither explained to be exclusively driven by data nor was it named a data-driven culture. Further, factors derived from the Analytics literature incompletely cover the organizational factors essential to enhance value from Analytics. Thus, Proposition 9 is rejected. However, the collected organizational factors strongly resemble the enhancing factors of knowledge integration of the KBV. First, common knowledge resembles organizational factors such as knowledge about available data to solve business problems ("data literacy"), understanding of the results and risks of analytical methods, and understanding (and appreciation) of the work behind Analytics. Second frequent and varying knowledge integration resembles two differing groups of organizational factors in the evidence. Frequency occurs through willingness to share data, willingness to use Analytics, emphasizing ("evangelizing") achieved and potential value from Analytics, and providing time to work with Analytics or on ideas for initiatives. The variability of knowledge integration occurs through value enhancing organizational factors of openness to ideas, creativity, curiosity, and openness to consider its own actions being possible sources of mistakes. However, the value of Analytics is enhanced by further organizational factors outside the scope of Analytics or the KBV's knowledge integration, such as willingness to cooperate, embracing change, holistic thinking, or a mindset fitting to the customer. These do not fit the proposition. Third, supporting structures resemble organizational structures for data sharing, structured processes of Analytics, infrastructure for Analytics, and alignment of Analytics with business goals. Interviewees recognized that not all factors can be present in an organization, but setting them as goals will lead an organization in a direction in which the value from Analytics is enhanced.

Rival Explanation 1 of Proposition 9-of all users needing to be data scientists or being comparably educated - was rejected as a doubtful future scenario, as already stated by Roßmann et al. (2018). Users need certain knowledge about Analytics to avoid fallacies, understand the work behind and implications of Analytics, and to create more rich ideas for business problems to tackle. However, this level is far from the abilities of data scientists. Interviewees anticipate an imminent rise of the need for users in business processes who are better educated on Analytics, but only to allow easier interaction with data scientists and for using Analytics solutions as part of their empowerment. The need for users to embrace innovation is far greater, which involves accepting data scientists as vital team members for collaboration along with other vital members.

The second rival explanation was rejected as well, since case studies clearly emphasized Analytics' value to be enhanced by the organizational culture. However, contrary to expectations, a culture is required that embraces collaborative thinking and acting, embraces innovation, and is open to change. An innovation culture was specifically and repeatedly mentioned, supplemented by an agile mindset, entrepreneurial mindset, rapid testing of ideas, and involvement of users and customers in design processes. 
Appropriate critical reviews of Analytics solutions are considered beneficial, and for some solutions, critical feedback from observing them in process provides improvement potential that is hard to achieve before deployment. As discussed above, engineering knowledge, a practiced way of thinking and doing business, or informed decision making based on older and simpler (analytical) methods can provide appropriate criticism and suggestions that should not be disregarded. However, an intuition-based culture should be avoided that ignores Analytics results, treats Analytics with disregard, and mistrust or rejects any form of change. Thus, Rival Explanation 3 is rejected. Mistrust or demand for unrealistic certainty levels of solutions will delay the use of solutions and result in missed opportunities.

In accordance with Proposition 5, an organizational factor in a large share of LSCM organizations is a missing appreciation for resources outside the physical "bread and butter" processes. Behind the leaders such as the case study organizations, there is a field of followers that treat IT as "hygiene factor", and which cannot build Analytics capabilities on that attitude.

In summary, all explanations, propositions and rivals have been rejected.

\subsection{The long-term usability of solution in Analytics initiatives}

An Analytics product meant for continuous use is not finished after deployment. It must be maintained and shows constant need for adjustments because of newly available data and data sources, needs and feedback from users, degradation of solution performance over time, and evaluation of achieved business outcome. As part of deployment and as described above, there is usually an adjustment phase of the solution to the business process. Maintenance comes after this adjustment and is necessary to assure performance through monitoring and quality control ("Analytics on Analytics "), and adjustments to changes in the technological ecosystem (e.g., the solution hosting system or systems the solution is interacting with). Further, maintenance involves extending existing solutions by including changes present in more recent data like new patterns, new anomalies, or different behavior of processes and customers triggered by the Analytics solution or market and societal changes, which shift optimal points and priorities of recommended actions. Solutions are likely transferred or scaled with smaller changes to new user groups with similar business problems. Further, solutions will be overhauled or replaced if new ideas or newly tested analytical methods on solving problems migrate from more recent initiatives to the focal solution or if the promoted and visible value and benefits from a solution induce demand for additional features or ideas for improvement. Interviewees observed a surge of new ideas for Analytics initiatives once users had been exposed to helpful Analytics solutions and emphasized the ongoing opportunities for improvement in organizations, which can be supported by Analytics. This resembles the continuous renewal of capabilities to sustain competitive advantage specified in the KBV of extending the capabilities to include new knowledge and using existing knowledge in new capabilities. This provides support for Proposition 10. However, discoveries usually experience little to no maintenance. 
Rival Explanation 1 of Proposition 10, which states that Analytics solutions are stable and, thus, represents the opposite of Proposition 10, is rejected. As discussed above, Analytics solutions need regular adjustments to internal and external developments along the solutions' lifecycles. A multitude of aspects of solutions can change including input, functionality, or the business and technological environments of the solutions, which will influence their performance.

Analytics solutions will not be adapted to individual users. Solutions are developed for certain roles, and if roles do not change in objective criteria or tasks, resulting in a change of users' behaviors, there is no need for user-based adjustments. Thus, Rival Explanation 2 is rejected. If new users are introduced to an Analytics solution, because the solution is newly developed or the users newly hired, they will usually be trained. Too much individualization creates chaos, but there should be room for personalization for consumability. However, users' requests will be considered for solution improvement and building the trust of users. But these considerations include prioritization and removal of features that turned out bad.

Finally, Rival Explanation 3 of solution replacement by newly available technologies is rejected. New technologies will not automatically trigger adjustments. The choice of technologies is driven by the business problems and a change of technologies for solving a business problem requires justification by improved performance or fit to requirements, exceeding the cost of migrating to the technology. Migrating solutions to new and appropriate technologies is likely if existing technologies present a bottleneck. In addition, a technology's cost-benefit evaluation changes as the technology matures, becoming less costly or addressing more needs. Cost evaluation should additionally be done for the long term and with anticipation of future requirements to stay on track with the competition.

Regarding the decentralization of LSCM functions due to globally operating supply chains, interviewees emphasized the need to have a vision about scaling a solution in development to similar supply chain functions in other locations after it has been tested and validated. This should avoid these functions having redundant development efforts or creating isolated solutions.

In summary, Proposition 10 is supported, and all rivals are rejected.

\section{Conclusion}

This research has developed an explanation of how Analytics generates competitive advantage for organizations based on the knowledge-based view and using the example of Logistics and Supply Chain Management in Germany, as illustrated in Fig. 10. In short, managers need to identify business problems with tangibly beneficial solutions that are complex or business critical. They select expertise, creativity, and data from internal experts and acquire it from experts from the market, and manage the experts to find the solution. Within the bounds of a structured initiative, this team of cross-functional experts solves the problem experimentally and iteratively by presenting intermediate insights extracted from data to users for evaluation and directions. If the intermediate insights fulfill the 


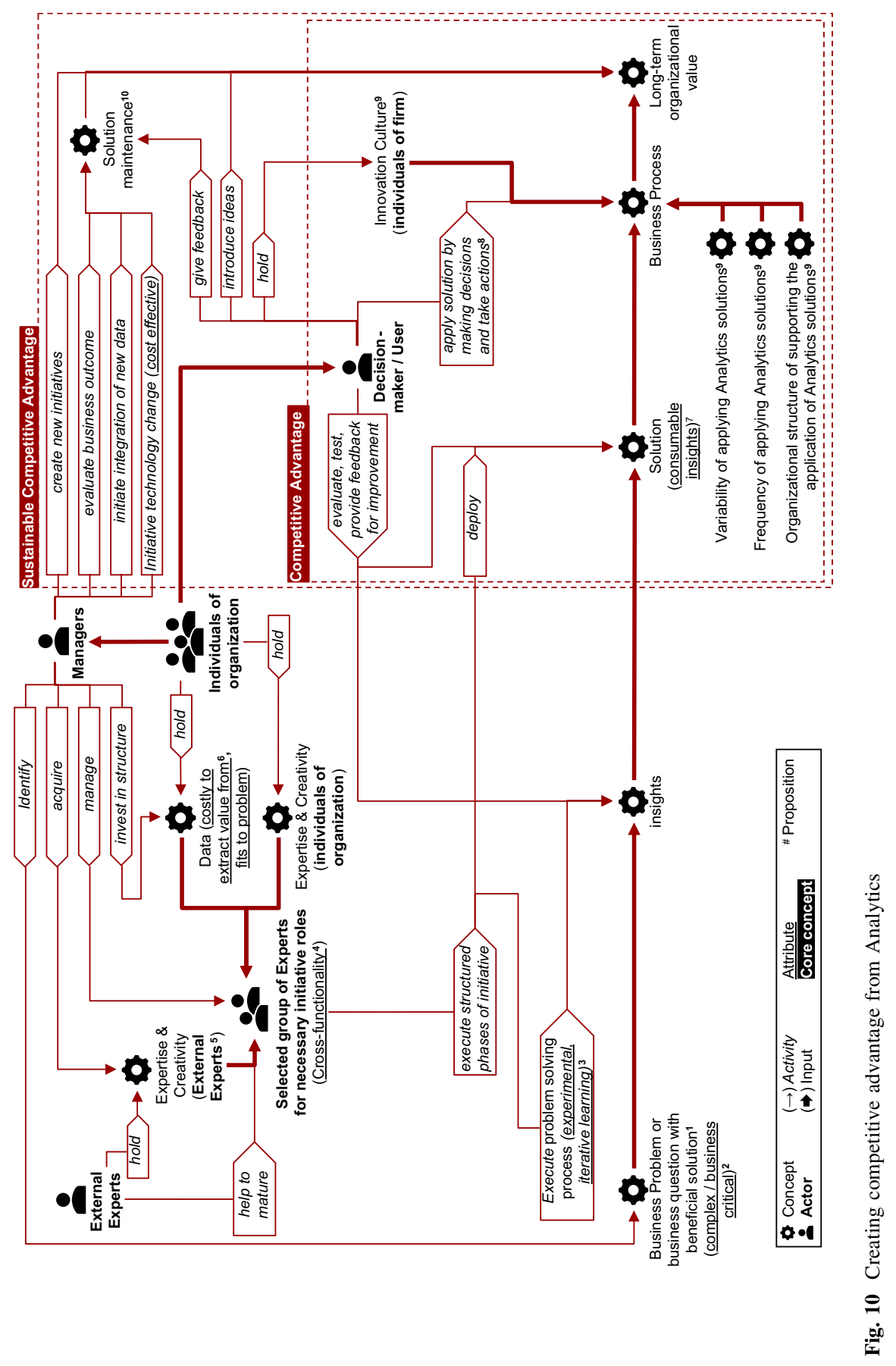


performance criteria of the users, a consumable solution is developed that is appropriate to the decision making of users and the process requirements for decision making. The users must integrate the solution into their decision-making process during value creation in the business process and make decisions and take actions accordingly. The impact of integrating Analytics into the value creation process is enhanced by the variability of applying Analytics solutions, the frequency of their use, and supporting organizational structures, as well as a culture fostering innovation and welcomes change. Analytics solutions, which are created to solve complex and business critical problems that are ahead of the problems the competition has solved and are applied frequently and amongst a variety of such solutions in the value creation process, provide value that has the potential to generate competitive advantage to an organization. To sustain such competitive advantage, users need to provide feedback on deployed Analytics solutions and introduce further ideas for new solutions. Based on the feedback, evaluation of business outcomes, and collection of new data, managers must initiate maintenance and advancement of deployed solutions. Further, they must create new Analytics initiatives from the ideas.

Hence, Analytics presents a manifestation of knowledge-based generation of competitive advantage. This research emphasizes it as supplement to the organizations' toolboxes that is suitable for problems with relevant knowledge held in data or for which data can be collected-which are becoming more and more common. It does not present a single standing way of generating competitive advantage for most organizations. As with any tool, the value generated from it depends on the right use and, further, it does not guarantee competitive advantage.

This explanation was concluded from eight confirmatory case studies, which provided evidence to support or reject propositions based on the resemblance of the $\mathrm{KBV}$ with the Analytics literature and rival explanations to these propositions. The extensive data collection and analysis resulted in eight propositions being supported, one proposition being rejected because of an incomplete explanation, and another proposition being rejected. However, due to the research design, the collected evidence allowed to derive an explanation of how competitive advantage can be generated from Analytics. The rival explanations, mostly rejected, nevertheless supported the explanation by adjustments and demonstrations of exceptions to shape the explanation.

While the research has been conducted in the domain of LSCM for methodological reasons, it is a reasonable assumption that the results apply to other domains in a comparable manner. Regarding the presented results and interviewees' comments on incorporating information technology organizations and expert organizations on Analytics, these organizations development processes and aims for novel Analytics solutions are likely to differ to a certain degree. Instead of using their results exclusively to provide or improve their services and products, these organizations also intend to sell their results (solutions or expertise) on the market. For other domains, Analytics is likely to present another tool in the toolbox to create value that should be used if it promises superior results over other tools. In that manner, the process described above represents a promising blueprint for Analytics initiatives in other domains as well. 


\subsection{Theoretical implications}

This research contributes to two research streams. It provides supporting evidence for the validity of the research stream of the knowledge-based view due to its manifestation as the lifecycle of Analytics initiatives. The necessity of not just holding resources but using them in the right way in the value creation process is strongly supported by the evidence. Thus, any technology and concept must be evaluated on the use cases it provides and the problems it can solve in organizations. It should not be hyped for a potential value that cannot migrate into the processes of organizations. The importance of the effort of integrating knowledge into the value creation process, the role of individuals in the organization, and of the integration process as formulated in the KBV are strongly supported by the evidence. The moderating factors are prone to be overlooked in consideration of the necessary characteristics of the resources.

The role of the market as knowledge source was discovered to be broader than described in either the KBV or Analytics literature and should be investigated further. Research must take a deeper look at the changing role of the market in this digital economy. Valuable knowledge can be converted ever faster into microproducts and services and, thus, be sold to organizations, which can integrate the knowledge into their processes. Competitive advantage could be generated from a meaningful combination of market sourced micro-products and services to generate a unique and advantageous value creation process.

For the research streams of Analytics, a theoretical foundation for the effectiveness of best practices has been provided. This foundation provides explanations for the superiority of certain practices, which are experienced as working better than others. Further, an emphasis on cost effectiveness was introduced, presenting Analytics in the context of restrictions of mindful application and prioritization - to make data-driven decisions about the methods used for datadriven decision making. In addition, for this data-focused field that appears to be very technical, the reliance on creativity and ideas has been discussed and should receive further research attention regarding how to identify and support the right creative talent in organizations.

Regarding cross-functional problem solving with Analytics, the role of a mediator should be investigated further. While the role of translation between different experts has been declared as the mediator's limit, this interpretation might be undervalued. Further, this mediator might be specialized in methods to stimulate the generation of ideas and induce additional ideas from experts who hold specialized knowledge but are not using it at full capacity on a problem.

Research should further evaluate the use of agile methods for the research process. Short but frequent iterations with the audience the research wants to address might provide more impactful research to this audience. However, this is not intended as an argument for discarding scientific rigor, but a paradigm shift from thinking about how to address the audience, to a process of frequent exchange with the audience.

Regarding LSCM, this paper identifies the need for further research on the impact of digital and Analytics-based business models on LSCM. This paper has perceived 
a disregard of LSCM organizations for the risk from such business models, while they could eventually eat up profitable business and margins from LSCM organizations and force these organizations' services to a commodity status. This potential development should be studied further.

Finally, further research is necessary on the beneficial integration of visibility into LSCM processes and the changing decision landscape. While the visibility is increased, it may not be beneficial without the ability to exploit it and opportunities to act on it. To gain this ability, extensive change of the supply chain might be necessary.

\subsection{Managerial implications}

This research provides guidance for the execution of Analytics initiatives derived from the domain of LSCM, embodied in the subfield of supply chain Analytics. Managers are directed to base their pursuit for advantages, including competitive advantages, not just on analytical methods, but also on the problems chosen on which to use the analytical methods. To find these problems, managers must foster curiosity and the generation of ideas among employees. To ensure obtaining value from the solutions, they must build the trust of the intended users in the solutions and establish a culture that embraces change.

In this regard, this study advises not to glorify data scientists. While the role is new, scarce, and has potential to be impactful on organizations, it is strongly dependent on the other experts of the organization. An image of the data scientist as jack-of-all-trades who naturally supersedes the process experts by building datadriven solutions devalues these experts and negates their willingness to cooperate. Indeed, the cost-benefit ratio of such a jack-of-all-trades is a fallacy, because this quite expensive multi-talented individual has a finite workload capacity in which tasks outside mathematical and analytical specialization would have to be accommodated.

This study demonstrates that Analytics does not just comprise the analytical. Managers interested in using Analytics must be aware of iterative ways to build the solutions with cross-functional teams, especially including the intended users, to overcome the difficulty of extracting insights from the data and to ensure the deployment and use of the solution. They may contract providers to help them build maturity in Analytics but also for the purpose of getting access to knowledge they only need in rare instances and as an "extended workbench". Deployed solutions should be maintained to ensure performance and technical functionality and for improvements based on constant feedback and inspired by other initiatives.

There is a causality dilemma, since a good data infrastructure is required to identify beneficial use cases, but beneficial use cases are required to motivate investments into data infrastructure. Evidence suggests managers should make courageous investments in infrastructure to set a basis for valuable Analytics initiatives and to substantiate the desire to increase the use of Analytics in their organizations. Presenting and substantiating this desire is important to spark ideas for use cases and problems to tackle. As observed by the interviewees, asking the people in the processes and encouraging them to generate ideas will almost certainly 
result in Analytics initiatives that can create value in the organization and is the way to mature in Analytics and tackle more complex Analytics with a competitive edge.

Regarding LSCM, organizations which are already conducting projects in crossfunctional teams that bring all relevant functions to the table and work towards an end-to-end supply chain vision should consider exploiting this cross-functionality further. This study has highlighted the value of marginal knowledge, which can be operationalized by introducing additional experts into such cross-functional teams to generate new ideas and approaches.

This study emphasizes building a culture that supplements the strong pride in the physical process management and optimization skills with an appreciation of supporting functions such as IT and Analytics. This is certainly not routinely the case with market leaders, but the LSCM field is composed of a broad range of small and medium organizations, which can benefit from these supporting functions as well as need to be open to them.

\subsection{Future research and limitations}

Considering the evidence collected in this research, some aspects present a strong demand for future research. First, it is necessary to create guidance for data governance, investigate appropriate analytical actions that harm neither ethics nor privacy, systematize practices to ensure privacy while enabling analysis of organizational activities, and provide reflected discussions on ethical Analytics. Second, willingness to change is a long-lasting issue, which requires means for managers to investigate and overcome resistance to change in order for initiatives to be effective. Third, while the estimated percentage of today's jobs likely to be automatable is very large, this research indicates that this ability might not be used, and control may be kept in the hands of users. Research is necessary to understand which decisions managers will not give out of hand and for what reason, to provide a better projection of the future of automation and allow a longitudinal observation to reveal whether these reasons are sustained or change over time.

There are limitations that apply to this research. This research was conducted on the example of LSCM and, consequently, provides a generalization within the bounds of the LSCM domain in Germany. As explained above, it is reasonable to assume that the results apply to other domains in a comparable manner. However, the collected evidence does not allow such interference. Hence, this research should be recreated for other domains, with an emphasis on the factors that distinguish each domain in the use of Analytics. Further, there has been limited access to interviewees, which has been compensated with an extensive review of documents. The number of experts available for this investigation was limited due to the novelty of Analytics to a broader range of domains and organizations. Further, these experts are busy and work on topics for which disclosure is undesired. In addition, the number of case studies could have been increased for increased validity. Finally, while confirmatory case studies are rarely observed in the scientific literature as opposed to survey research for collecting confirmatory evidence, the approach was chosen to gain the opportunity to shape the explanation in focus. Thus, further quantitative confirmatory methods might be employed to validate the results. 
Open Access This article is distributed under the terms of the Creative Commons Attribution 4.0 International License (http://creativecommons.org/licenses/by/4.0/), which permits unrestricted use, distribution, and reproduction in any medium, provided you give appropriate credit to the original author(s) and the source, provide a link to the Creative Commons license, and indicate if changes were made.

\section{References}

Acito, Frank, and Vijay Khatri. 2014. Business Analytics: Why now and what next? Business Horizons 57(5): 565-570. Kelley School of Business, Indiana University. https://doi.org/10.1016/j.bushor. 2014.06.001.

Alavi, Maryam, and Dorothy E. Leidner. 2001. Review: Knowledge management and knowledge management systems: conceptual foundations and research issues. MIS Quarterly 25: 107. https:// doi.org/10.2307/3250961.

Alles, B. Y. David, and John Burshek. 2016. Ranking Analytics maturity by industry. International Institutefor Analytics.

Amit, Raphael, and Paul J.H. Schoemaker. 1993. Strategic assets and organizational rent. Strategic Management Journal 14: 33-46. https://doi.org/10.1002/smj.4250140105.

Barney, Jay B. 1986. Strategic factor markets: expectations, luck, and business strategy. Management Science 32: 1231-1241. https://doi.org/10.1287/mnsc.32.10.1231.

Barney, Jay B. 1991. Firm resources and sustained competitive advantage. Journal of Management 17: 99-120. https://doi.org/10.1177/014920639101700108.

Barton, Dominic, and David Court. 2012. Making advanced Analytics work for you. Harvard Business Review 90: 78-83. https://doi.org/10.1007/978-3-642-49298-3.

Beer, David. 2018. Envisioning the power of data Analytics. Information, Communication \& Society 21: 465-479. https://doi.org/10.1080/1369118x.2017.1289232.

Bose, Ranjit. 2009. Advanced Analytics: opportunities and challenges. Industrial Management \& Data Systems 109: 155-172. https://doi.org/10.1108/02635570910930073.

Bowersox, Donald J., David J. Closs, and M. Bixby Cooper. 2007. Supply chain logistics management. Boston: McGraw-Hill/Irwin.

Brinch, Morten, Jan Stentoft, Jesper Kronborg Jensen, and Christopher Rajkumar. 2018. Practitioners understanding of big data and its applications in supply chain management. The International Journal of Logistics Management 29: 555-574. https://doi.org/10.1108/ijlm-05-2017-0115.

Cady, Field. 2017. The data science handbook. Hoboken, NJ: Wiley.

Canonico, Paolo, Ernesto De Nito, and Gianluigi Mangia. 2012. Control mechanisms and knowledge integration in exploitative project teams: a case study from the coal fired power plant industry. Edited by Giovanni Schiuma. Journal of Knowledge Management 16: 538-549. https://doi.org/10. $1108 / 13673271211246121$.

Cao, Guangming, Yanqing Duan, and Gendao Li. 2015. Linking business Analytics to decision making effectiveness: A Path model analysis. IEEE Transactions on Engineering Management 62: 384-395.

Capgemini. 2015. Big \& fast data: The rise of insight-driven business.

Carillo, Kevin Daniel André. 2017. Let's stop trying to be "sexy"-preparing managers for the (big) data-driven business era. Business Process Management Journal 23: 598-622. https://doi.org/10. 1108/bpmj-09-2016-0188.

Chae, Bongsug, David Olson, and Chwen Sheu. 2014a. The impact of supply chain Analytics on operational performance: a resource-based view. International Journal of Production Research 52: 4695-4710. https://doi.org/10.1080/00207543.2013.861616.

Chae, Bongsug, Chenlung Yang, David Olson, and Chwen Sheu. 2014b. The impact of advanced Analytics and data accuracy on operational performance: A contingent resource based theory (RBT) perspective. Decision Support Systems 59: 119-126. https://doi.org/10.1016/j.dss.2013.10.012.

Chavez, Roberto, Yu. Wantao, Mark A. Jacobs, and Mengying Feng. 2017. Data-driven supply chains, manufacturing capability and customer satisfaction. Production Planning \& Control 28: 906-918. https://doi.org/10.1080/09537287.2017.1336788. 
Chen, Hsinchun, Roger H.L. Chiang, and Veda C. Storey. 2012. Business intelligence and Analytics: from big data to big impact. MIS Quarterly 36: 1165-1188. https://doi.org/10.1007/978-1-46141557-2.

Choo, Adrian S., Rajiv Nag, and Yusen Xia. 2015. The role of executive problem solving in knowledge accumulation and manufacturing improvements. Journal of Operations Management 36: 63-74. https://doi.org/10.1016/j.jom.2015.03.001.

Conner, Kathleen R., and C.K. Prahalad. 1996. A resource-based theory of the firm: Knowledge versus opportunism. Organization Science 7: 477-501. https://doi.org/10.1287/orsc.7.5.477.

Cui, Miao, and Shan L. Pan. 2015. Developing focal capabilities for e-commerce adoption: A resource orchestration perspective. Information \& Management 52: 200-209. https://doi.org/10.1016/j.im. 2014.08.006.

Davenport, Thomas H. 2009. Realizing the potential of retail Analytics-plenty of food for those with the appetite. MA: Babson Park.

Davenport, Thomas H. 2013. Analytics 3.0. Harvard Business Review 91: 64-72.

Davenport, Thomas H., and Jeanne G. Harris. 2007. Competing on Analytics: The new science of winning. Boston: Harvard Business School Press. https://doi.org/10.1225/3323.

Davenport, Thomas H., Jeanne G. Harris, David W. De Long, and Alvin L. Jacobson. 2001. Data to knowledge to results. California Management Review 43: 117.

Demsetz, Harold. 1988. The theory of the firm revisited. Journal of Law Economics and Organization 4: $141-161$.

Dyer, Jeffrey H., and Harbir Singh. 1998. The relational view: Cooperative strategy and sources of interorganizational competitive advantage. The Academy of Management Review 23: 660. https:// doi.org/10.2307/259056.

Eisenhardt, Kathleen M. 1989. Building Theories from Case Study Research. The Academy of Management Review. 14 (4): 532-550.

Franks, Bill. 2014. The Analytics revolution: How to improve your business by making Analytics operational in the big data Era. New York: John Wiley \& Sons.

Galbraith, Jay R. 1973. Designed complex organizations. Reading, Mass: Addison-Wesley.

Gandomi, Amir, and Murtaza Haider. 2015. Beyond the hype: Big data concepts, methods, and Analytics. International Journal of Information Management 35: 137-144. https://doi.org/10.1016/j.ijinfomgt. 2014.10.007.

Gavetti, Giovanni, and Daniel Levinthal. 2000. Looking forward and looking backward: cognitive and experiential search. Administrative Science Quarterly 45: 113. https://doi.org/10.2307/2666981.

Gerring, John, and Lee Cojocaru. 2016. Selecting cases for intensive analysis. Sociological Methods \& Research 45: 392-423. https://doi.org/10.1177/0049124116631692.

Ghasemaghaei, Maryam, Khaled Hassanein, and Ofir Turel. 2017. Increasing firm agility through the use of data Analytics: The role of fit. Decision Support Systems 101: 95-105. https://doi.org/10.1016/j. dss.2017.06.004.

Grant, Robert M. 1996a. Toward a knowledge-based theory of the firm. Strategic Management Journal 17: 109-122. https://doi.org/10.2307/2486994.

Grant, Robert M. 1996b. Prospering in dynamically-competitive environments: organizational capability as knowledge integration. Organization Science 7: 13. https://doi.org/10.1287/orsc.7.4.375.

Guerra, Peter, and Kirk Borne. 2016. Ten signs of data science maturity. Sebastopol: O'Reilly Media.

Heugens, Pursey P.M.A.R., Cees B.M. van Riel, and Frans A.J. Van Den Bosch. 2004. Reputation management capabilities as decision rules. Journal of Management Studies 41: 1349-1377. https:// doi.org/10.1111/j.1467-6486.2004.00478.x.

Holsapple, Clyde, Anita Lee-Post, and Ram Pakath. 2014. A unified foundation for business Analytics. Decision Support Systems 64: 130-141.

Hsieh, Chihmao, Jack A. Nickerson, and Todd R. Zenger. 2007. Opportunity discovery, problem solving and a theory of the entrepreneurial firm. Journal of Management Studies 44: 1255-1277. https://doi. org/10.1111/j.1467-6486.2007.00725.x.

Hurnonen, Salla, Paavo Ritala, and Hanna-Kaisa Ellonen. 2016. The role of knowledge-integration practices in service innovation projects. International Journal of Innovation Management 20: 1650007. https://doi.org/10.1142/s1363919616500079.

Janssen, Marijn, Haiko van der Voort, and Agung Wahyudi. 2017. Factors influencing big data decisionmaking quality. Journal of Business Research 70: 338-345. https://doi.org/10.1016/j.jbusres.2016. 08.007 . 
Jeppesen, Lars Bo, and Karim R. Lakhani. 2010. Marginality and problem-solving effectiveness in broadcast search. Organization Science 21: 1016-1033. https://doi.org/10.1287/orsc.1090.0491.

Jeske, Martin, Mortiz Grüner, and Frank Weiß. 2013. Big data in logistics. Troisdorf: DHL Customer Solutions \& Innovation.

Kersten, W., M. Seiter, B. von See, N. Hackius, and T. Maurer. 2017. Trends und strategien in logistik undsupply chain management-Chancen der digitalen Transformation. Hamburg: DVV Media Group.

Kiron, David, Pamela Kirk Prentice, and Renee Boucher Ferguson. 2014. Raising the bar with Analytics. MIT Sloan Management Review 55: 28-33.

Kiron, David, Rebecca Shockley, Nina Kruschwitz, Glenn Finch, and Michael Haydock. 2012. Analytics : The widening divide. MIT Sloan Management Review 53: 1-23.

Kogut, Bruce, and Udo Zander. 1992. Knowledge of the firm, combinative capabilities, and the replication of technology. Organization Science 3: 383-397. https://doi.org/10.1287/orsc.3.3.383.

Lai, Yuanyuan, Huifen Sun, and Jifan Ren. 2018. Understanding the determinants of big data Analytics (BDA) adoption in Logistics and Supply Chain Management. The International Journal of Logistics Management 29: 676-703. https://doi.org/10.1108/ijlm-06-2017-0153.

Larson, Deanne, and Victor Chang. 2016. A review and future direction of agile, business intelligence, Analytics and data science. International Journal of Information Management 36: 700-710. https:// doi.org/10.1016/j.ijinfomgt.2016.04.013.

Lavalle, Steve, Eric Lesser, Rebecca Shockley, Michael S. Hopkins, and Nina Kruschwitz. 2011. Big data, Analytics and the path from insights to value. MIT Sloan Management Review 52: 21-32. https://doi.org/10.0000/pmid57750728.

Leventhal, Barry. 2015. All models are wrong but some are useful: the use of predictive Analytics in direct marketing. Quality Technology \& Quantitative Management 12: 93-104. https://doi.org/10. 1080/16843703.2015.11673369.

Lewis, Michael, Alistair Brandon-Jones, Nigel Slack, and Mickey Howard. 2010. Competing through operations and supply. International Journal of Operations \& Production Management 30: 1032-1058. https://doi.org/10.1108/01443571011082517.

Liberatore, Matthew J., and Wenhong Luo. 2010. The Analytics movement: implications for operations research. Interfaces 40: 313-324. https://doi.org/10.1287/inte.1100.0502.

Macher, Jeffrey T. 2006. Technological development and the boundaries of the firm: A knowledge-based examination in semiconductor manufacturing. Management Science 52: 826-843. https://doi.org/10. 1287/mnsc.1060.0511.

Macher, Jeffrey T., and Christopher Boerner. 2012. Technological development at the boundaries of the firm: a knowledge-based examination in drug development. Strategic Management Journal 33: 1016-1036. https://doi.org/10.1002/smj.1956.

Marchand, Donald A., and Joe Peppard. 2013. Why IT fumbles Analytics. Harvard Business Review 91: 104-112.

McAfee, Andrew, and Erik Brynjolfsson. 2012. Big data: the management revolution. Harvard business review 90: 60-68.

Miles, Matthew B., A. Michael Huberman, and Johnny Saldana. 2014. Qualitative data analysis: A methods sourcebook, 3rd ed. Thousand Oaks: SAGE.

Nickerson, Jack A., and Todd R. Zenger. 2004. A knowledge-based theory of the firm-the problem solving perspective. Organization Science 15: 617-632. https://doi.org/10.1287/orsc.1040.0093.

Nonaka, Ikujiro, and Georg von Krogh. 2009. Perspective-tacit knowledge and knowledge conversion: controversy and advancement in organizational knowledge creation theory. Organization Science 20: 635-652. https://doi.org/10.1287/orsc.1080.0412.

Provost, Foster, and Tom Fawcett. 2013. Data science for business. Sebastopol: O'Reilly Media.

Purvis, Russell L., V. Sambamurthy, and Robert W. Zmud. 2001. The assimilation of knowledge platforms in organizations: an empirical investigation. Organization Science 12: 117-135. https:// doi.org/10.1287/orsc.12.2.117.10115.

Ransbotham, Sam, David Kiron, and Pamela Kirk Prentice. 2015. Minding the Analytics gap. MIT Sloan Management Review 56: 63-68.

Rosenbaum, Paul R. 2002. Observational studies. Observational studies. Springer Series in Statistics, 2nd ed. New York: Springer.

Ross, Jeanne W., Cynthia M. Beath, and Anne Quaadgras. 2013. You may not need big data after all. Havard Business Review 91: 90-98. 
Roßmann, Bernhard, Angelo Canzaniello, Heiko von der Gracht, and Evi Hartmann. 2018. The future and social impact of big data Analytics in supply chain management: Results from a Delphi study. Technological Forecasting and Social Change 130: 135-149. https://doi.org/10.1016/j.techfore. 2017.10.005.

Sanders, Nada R. 2016. How to use big data to drive your supply chain. California Management Review 58: 26-48. https://doi.org/10.1525/cmr.2016.58.3.26.

Schoenherr, Tobias, and Cheri Speier-Pero. 2015. Data science, predictive Analytics, and big data in supply chain management: current state and future potential. Journal of Business Logistics 36: 120-132. https://doi.org/10.1111/jbl.12082.

Seddon, Peter B., Dora Constantinidis, Toomas Tamm, and Harjot Dod. 2017. How does business Analytics contribute to business value? Information Systems Journal 27: 237-269. https://doi.org/ 10.1111/isj.12101.

Seuring, Stefan A. 2008. Assessing the rigor of case study research in supply chain management. Supply Chain Management: An International Journal 13: 128-137. https://doi.org/10.1108/ 13598540810860967.

Simchi-Levi, David, Edith Simchi-Levi, and Philip Kaminsky. 2003. Designing and managing the supply chain: Concepts, strategies, and cases, 3rd ed. Boston: McGraw-Hill New York.

Simon, Herbert A. 1962. The Architecture of Complexity. Proceedings of the American Philosophical Society 106: 467-482.

Simon, Herbert A. 1973. The structure of ill structured problems. Artificial Intelligence 4: 181-201. https://doi.org/10.1016/0004-3702(73)90011-8.

Souza, Gilvan C. 2014. Supply chain Analytics. Business Horizons 57: 595-605. https://doi.org/10.1016/ j.bushor.2014.06.004.

Spanos, Yiannis E., and Gregory Prastacos. 2004. Understanding organizational capabilities: towards a conceptual framework. Journal of Knowledge Management 8: 31-43. https://doi.org/10.1108/ 13673270410541024.

Spender, J.-C. 1996. Making knowledge the basis of a dynamic theory of the firm. Strategic Management Journal 17: 45-62. https://doi.org/10.1002/smj.4250171106.

Srinivasan, Ravi, and Morgan Swink. 2018. An investigation of visibility and flexibility as complements to supply chain Analytics: An organizational information processing theory perspective. Production and Operations Management 27: 1849-1867. https://doi.org/10.1111/poms.12746.

Strauss, Anselm L., and Juliet M. Corbin. 1998. Basics of qualitative research: techniques and proceduresfor developing grounded theory. London: SAGE.

Teece, David J. 1981. The market for know-how and the efficient international transfer of technology. The ANNALS of the American Academy of Political and Social Science 458: 81-96. https://doi.org/ $10.1177 / 000271628145800107$.

Teece, David J. 1998. Capturing value from knowledge assets. California Management Review 40: 55-79.

Thieullent, Anne-Laure, Mathieu Colas, Jerome Buvat, K.V.J. Subrahmanyam, and Ashish Bisht. 2016. Going Big: Why Companies Need to Focus on Operational Analytics. Paris: Capgemini.

Tiwana, Amrit. 2008. Do bridging ties complement strong ties? An empirical examination of alliance ambidexterity. Strategic Management Journal 29: 251-272. https://doi.org/10.1002/smj.666.

Trkman, Peter. 2010. The critical success factors of business process management. International Journal of Information Management 30: 125-134. https://doi.org/10.1016/j.ijinfomgt.2009.07.003.

Trkman, Peter, Kevin McCormack, Marcos Paulo Valadares de Oliveira, and Marcelo Bronzo Ladeira. 2010. The impact of business Analytics on supply chain performance. Decision Support Systems 49: 318-327. https://doi.org/10.1016/j.dss.2010.03.007.

Vachon, Stephan, and Robert D. Klassen. 2008. Environmental management and manufacturing performance: The role of collaboration in the supply chain. International Journal of Production Economics 111: 299-315. https://doi.org/10.1016/j.ijpe.2006.11.030.

De Ven, Van, H. Andrew, Andre L. Delbecq, and Richard Koenig. 1976. Determinants of coordination modes within organizations. American Sociological Review 41: 322-338. https://doi.org/10.2307/ 2094477.

Viaene, S., and A. Van den Bunder. 2011. The secrets to managing business Analytics projects. MIT Sloan Management Review 53: 65-69.

Voss, Chris, Nikos Tsikriktsis, and Mark Frohlich. 2002. Case research in operations management. International Journal of Operations and Production Management 22: 195-219. https://doi.org/10. 1108/01443570210414329. 
Waller, Matthew A., and Stanley E. Fawcett. 2013. Data science, predictive Analytics, and big data: A revolution that will transform supply chain design and management. Journal of Business Logistics 34: 77-84. https://doi.org/10.1111/jbl.12010.

Wang, Ying, Filip Caron, Jan Vanthienen, Lei Huang, and Yi Guo. 2014. Acquiring logistics process intelligence: Methodology and an application for a Chinese bulk port. Expert Systems with Applications 41: 195-209. https://doi.org/10.1016/j.eswa.2013.07.021.

Watson, Hugh J. 2014. Tutorial: big data Analytics: concepts, technologies, and applications. Communications of the Association for Information Systems 34: 1247-1268.

Winter, Sidney G. 1987. Knowledge and competence as strategic assets. The competitive Challange, 159-184. David J: Teece.

Wixom, Barbara H., Bruce Yen, and Michael Relich. 2013. Maximizing value from business Analytics. MIS Quarterly Executive 12: 111-123.

Yin, Robert K. 2014. Case study research: Design and methods. London: SAGE Publications Ltd.

Zack, Michael H. 1999. Developing a knowledge strategy. California Management Review 41: 125-145. https://doi.org/10.2307/41166000.

Publisher's Note Springer Nature remains neutral with regard to jurisdictional claims in published maps and institutional affiliations. 